\title{
Structural Econometric Methods in Auctions: A Guide to the Literature*
}

forthcoming, Journal of Econometric Methods (inaugural issue), Berkley Electronic Press

\author{
Brent R. Hickman ${ }^{\dagger}$ \\ Timothy P. Hubbard ${ }^{\ddagger}$ \\ Yiğit Sağlam ${ }^{\S}$
}

\begin{abstract}
Auction models have proved to be attractive to structural econometricians who, since the late 1980s, have made substantial progress in identifying and estimating these rich game-theoretic models of bidder behavior. We provide a guide to the literature in which we contrast the various informational structures (paradigms) commonly assumed by researchers and uncover the evolution of the field. We highlight major contributions within each paradigm and benchmark modifications and extensions to these core models. Lastly, we discuss special topics that have received substantial attention among auction researchers in recent years, including auctions for multiple objects, auctions with risk averse bidders, testing between common and private value paradigms, unobserved auction-specific heterogeneity, and accounting for an unobserved number of bidders as well as endogenous entry.
\end{abstract}

Keywords: auctions, structural econometrics.

JEL Classification Numbers: C01, D44.

\footnotetext{
*We were all students of Harry J. Paarsch and would like to express our sincere gratitude and appreciation to him. Harry was not only a pioneer of this literature, but he introduced each of us to the field and continues to inspire us today. We would also like to thank two anonymous referees for helpful suggestions. Any errors are our own.

${ }^{\dagger}$ Department of Economics, University of Chicago, 1126 E. 59 $9^{\text {th }}$ St., Chicago, IL 60637, USA. E-mail: hickmanbr@uchicago.edu

${ }^{\ddagger}$ Department of Economics, Texas Tech University, P.O. Box 41014, Lubbock, TX 79409, USA. E-mail: timothy.hubbard@ttu.edu

${ }^{\S}$ School of Economics and Finance, Victoria University of Wellington, RH 312 Rutherford House, 23 Lambton Quay, Wellington, New Zealand. E-mail: yigit.saglam@vuw.ac.nz
} 


\section{Introduction}

Over the past 15 years, a literature concerning the econometrics of auction data has emerged and quickly developed into one of the most successful areas of structural econometric research. Powerful methods of estimation and inference have been developed which have not only added to our understanding of human behavior, but have also provided useful tools for policy analysis. This survey is an attempt to uncover the evolution of auctions research that has used structural models and to catalog important contributions. Our focus on structural research is motivated by two important considerations. First, structural work allows for counterfactual studies of situations not observed in the data such as alternative pricing mechanisms or auction formats. Second, leveraging economic theory provides access to information that would not be accessible from the data alone such as efficiency of the observed allocations, bidder risk aversion, information rents, and dependence among bidders' private information.

In keeping our discussion general, we attempt only to provide a guide to the literature, so there will be less emphasis on the technical nuances of the works we discuss. Rather, our intent is to provide a broad synthesis of the various aspects of this sizeable literature. Our specific objectives of this survey are to identify and catalog the following five aspects of the field: (i) the modeling frameworks (informational paradigms) often employed; (ii) the types of questions these models are used to address; (iii) econometric identification of these models; (iv) general estimation methods used in bringing these models to real-world data; (v) types of data used and the general findings researchers have produced. A natural trade-off underlies our objectives: in allowing for breadth, we are forced to compromise the detail in which we discuss a particular piece of research. The interested reader is therefore directed to the original works for discussion of the econometric methods in their full splendor.

We have chosen to organize major contributions by partitioning the general affiliated values model model of auctions into subcategories based on commonly used informational structures (defined below) in hopes of complementing the book-length treatments of this topic in Paarsch and Hong (2006) or Athey and Haile (2007), who partitioned the literature by different auction formats. To our knowledge, no one has formally tied together the informational paradigms studied in empirical work in a unified way that is consistent with theoretical research. While our discussion of any one piece of research is no substitute for a thorough reading of the original work (we did not intend it to be), we have tried to provide enough insight into each piece of research we discussed so that readers can understand the fundamental contributions and have an understanding of how it fits in with previous and subsequent research. We think our discussions of the fundamental information paradigms not only provide a good introduction to researchers new to auctions and/or structural econometric methods, but that they also provide a perspective that will appeal to experienced researchers as well.

Auctions are typically modeled as games of incomplete information, so a primary concern to an auction researcher is the information structure; that is, how a given bidder receives and assimilates information about her own valuation(s) for the object(s) for sale as well as what each bidder 
knows concerning her rivals' valuations. Given that the econometrician's task in analyzing auction data involves characterizing the distribution over bidder heterogeneity, assumptions on the type of information structure will profoundly influence results and interpretation of empirical analyses. Therefore, we begin our survey by outlining a general framework proposed by Wilson (1977) as well as Milgrom and Weber (1982) which nests the various information structures that are encountered in the literature. We then use the various distinctions between environments to organize our guide to the literature on structural econometric research in auctions.

Another primary concern deals with the rules of the auction. Beginning with the work of Vickrey (1961), economists have systematically applied tools from game theory to investigate equilibrium behavior under four common formats in auctions for a single object: the oral, ascending-price or English auction; the oral, descending-price or Dutch auction; the first-price, sealed-bid auction; and the second-price, sealed-bid or Vickrey auction; as well as three common formats used in auctions for multiple objects: the sequential English auction, the uniform-price auction, and the discriminatory auction. Fundamental contributions to this effort were made by Wilson (1977, 1979) as well as Milgrom and Weber (1982) who, following the work of Harsanyi (1967), modeled auctions as games of incomplete information and adopted Bayesian-Nash equilibrium as the solution concept. Since then, the vast majority of empirical work in auctions has chosen to follow this approach. Equilibrium strategies, henceforth denoted by a function $\beta$, shall be briefly described in the various sections of the paper below, but only to the extent that will facilitate discussion of model identification and structural estimation. For a more in-depth discussion of the theory on equilibrium behavior in auctions, the interested reader is directed to excellent texts by Krishna (2002) and Milgrom (2004).

Given a set of data, a structural researcher must first identify which of these common auction formats apply. In addition to selecting an informational paradigm, a researcher must also specify whether bidders are ex-ante symmetric; that is, whether the marginal distributions of their private information (and/or their utility functions) are identical. After doing so, the researcher must argue that an equilibrium exists for the auction game of interest and, moreover, that one particular equilibrium uniquely corresponds to observed bidder behavior. Because structural researchers rely heavily on the model they adopt, this is where the work of auction theorists is most helpful. Proofs of existence and uniqueness are most often developed by theorists and are only related indirectly to the estimation process - they are beyond the scope of this survey. However, as our target audience will likely need to confront these issues at some point, we have included a table with a list of papers that deal with them in the Appendix.

This survey is organized as follows: in Section 2, we define the affiliated values paradigm and relate it to information structures that can be nested within this general framework. The four sections of the paper that follow explore how structural econometricians have identified and estimated these models using real-world auction data. In Section 7, we discuss special topics that have received substantial attention in recent years, including auctions for multiple objects, auctions with risk averse bidders, testing between common- and private-value paradigms, unobserved auctionspecific heterogeneity, and accounting for an unobserved number of bidders as well as endogenous 
entry. We conclude our survey in Section 8.

\section{Affiliated Values Paradigm}

Pioneering theoretical work on general models of auctions within the affiliated values (AV) paradigm was done by Wilson (1977) as well as Milgrom and Weber (1982).1. In what follows, we shall adopt notation that bridges these two seminal pieces. In the AV model, there are $\mathcal{N}$ bidders competing for an object. Bidders' valuations depend on a common component with a definite but unknown value $V$, and an idiosyncratic component, $S_{i}$. Bidder $i$ 's ex-post utility of consuming the object for sale is $u_{i}\left(s_{i}, v\right)$, where we make explicit our notational choice of denoting random variables by capital letters and realizations of these random variables by lowercase letters ${ }^{2}$ Similarly, in what follows we denote vector-valued variables in bold font and scalar-valued variables in standard mathematical font.

The general AV information structure has the following components:

- $S_{i}$ is private information to each bidder $i$.

- Conditional on $v$, the vector of signals $\mathbf{S}$ is viewed by all as a draw from a commonly known distribution, where

$$
\mathbf{S} \sim F_{\mathbf{S} \mid V}(\mathbf{s} \mid v)
$$

with corresponding density $f_{\mathbf{S} \mid V}$ and non-negative support $[\underline{s}, \bar{s}]^{\mathcal{N}}$. Conditioning on $v$ here provides greater generality, but it also forms an intuitive link between the theory and many real-world phenomena. For example, Hendricks, Pinkse, and Porter (2003) study oil lease auctions in which, prior to bidding, oil firms independently hire geological consultants to estimate the mineral content of a certain tract of ocean floor. In this case, the random variable $V$ is (a function of) the actual mineral content, and $S_{i}$ is the estimate of $V$ resulting from the $i^{\text {th }}$ geological survey. It is natural in this case to assume that the distribution of $S$ varies with each realization $v$.

- As $V$ is unknown, it is modeled as a random draw from a distribution

$$
V \sim F_{V}(v)
$$

with corresponding density $f_{V}$ and non-negative support $[\underline{v}, \bar{v}]$.

\footnotetext{
${ }^{1}$ Our focus in this section, and, throughout most of the paper is on single-object auctions. However, the informational structures we discuss extend intuitively (but with substantially more complexity) to the multi-object setting, which we discuss in Section 7.

${ }^{2}$ Whenever we use the terms ex ante or ex post, they refer to the point in time when bidding takes place. Thus, the ex-post utility of the winner is her utility of consuming the object after bidding has concluded. A bidder's ex-ante utility is her expected ex-post utility.
} 
- In general, all information contained in $F_{\mathbf{S} \mid V}$ and $F_{V}$ can be summarized by a joint distribution

$$
(\mathbf{S}, V) \sim F_{\mathbf{S}, V}(\mathbf{S}, V)
$$

with corresponding affiliated joint density $f_{\mathbf{S}, V}$ having support $[\underline{s}, \bar{s}]^{\mathcal{N}} \times[\underline{v}, \bar{v}]$.

- The $S_{i}$ 's are affiliated random variables (hence the name "affiliated values"). Briefly, stochastic affiliation is a strong form of positive correlation, and is defined as a set of random variables $\left(S_{1}, S_{2}, \ldots, S_{\mathcal{N}}\right)$ having joint density function $f$ which is log-supermodular; that is, $f$ satisfies $f\left(\mathbf{S} \vee \mathbf{S}^{\prime}\right) f\left(\mathbf{S} \wedge \mathbf{S}^{\prime}\right) \geq f(\mathbf{S}) f\left(\mathbf{S}^{\prime}\right)$, where $\vee(\wedge)$ denotes the coordinatewise minimum (maximum) among vectors $\mathbf{S}$ and $\mathbf{S}^{\prime}$. When $f$ is twice continuously differentiable, then equivalently,

$\left(S_{1}, \ldots, S_{\mathcal{N}}\right)$ are affiliated if and only if for all $i \neq j, \frac{\partial^{2}}{\partial s_{i} \partial s_{j}} \ln f \geq 0$, in which case $f$ is said to be affiliated. For a discussion of stochastic affiliation and its implications for auctions, see Section 3 as well as the Appendix of Milgrom and Weber (1982) or Appendix D of Krishna (2002). It should be noted that, although affiliation only infrequently arises explicitly as part of discussion in structural models of auctions, it arises implicitly in the majority of the papers we shall discuss: until only recently, all theory on existence and uniqueness of equilibria in Dutch and first-price auctions was based on the assumption of affiliation (see Govindan and Wilson (2010a b) and de Castro (2010) for recent departures from this norm).

The general AV model is often partitioned into two branches: the common values (CV) paradigm and the affiliated private values (APV) paradigm. However, some researchers have adopted another information structure which overlaps both CV and APV structures: the conditionally independent private information (CIPI) paradigm. In Figure 1, we present a visual representation of this partition of the AV framework along with some important subcases. In the subsections that follow, we discuss the CV, APV, and CIPI models in more detail with particular emphasis on relating the models to the informational structure we've outlined above. One drawback of the general AV paradigm is that distinctions between its two main branches are not testable empirically. In particular, Laffont and Vuong (1996) showed that any CV model is observationally equivalent to some APV model. For this reason, estimation within the general AV paradigm has been sparse. Moreover, this result implies that additional sources of model variation are needed in order to test between the two main branches of the AV family of models, such as (exogenously) varying numbers of bidders across different auctions.

\subsection{Common Values Paradigm}

The CV paradigm, also known as the mineral rights model or the interdependent values model, is a special case of $\mathrm{AV}$ in which $S_{i}$ does not perfectly reflect the intrinsic worth of the object for sale, but rather, it is just a noisy signal of value. That is, ex-post utility is given by

$$
u_{i}\left(s_{i}, v\right)=u_{i}(v)
$$


AFFILIATED VALUES

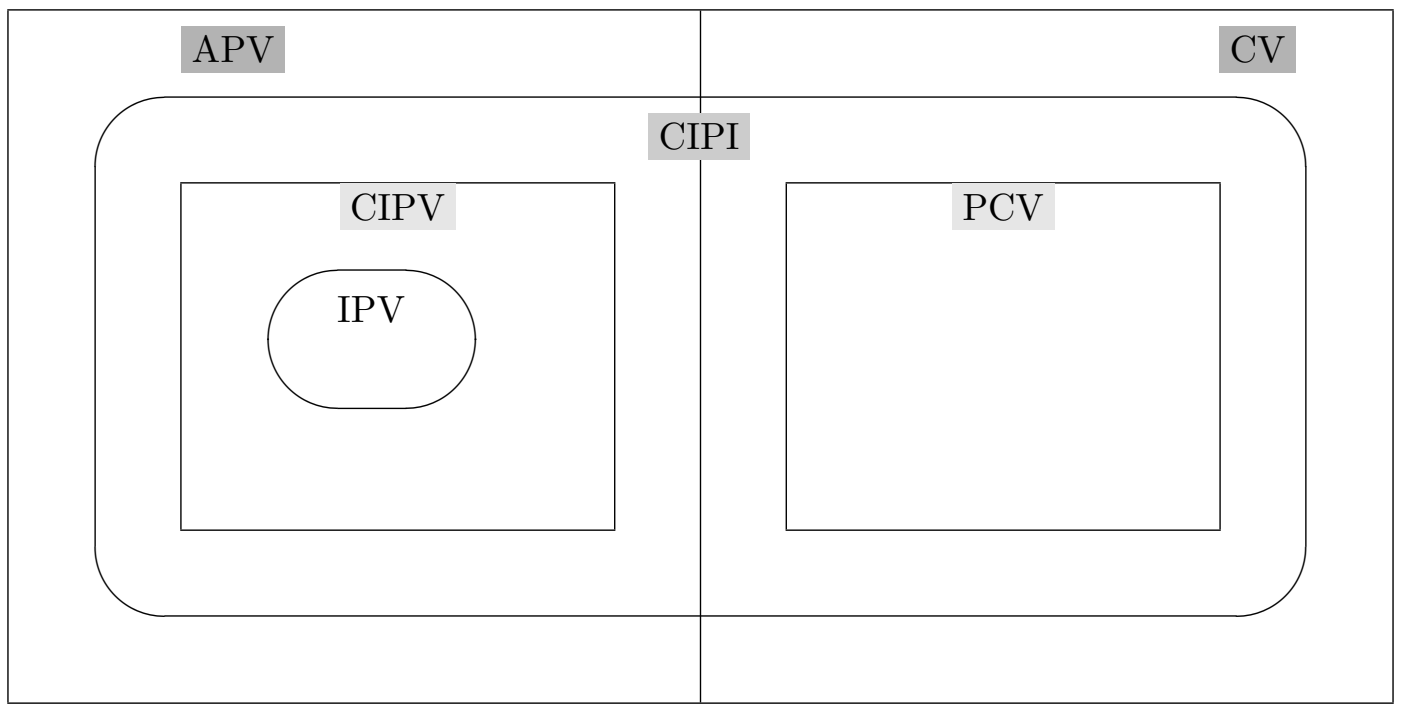

Figure 1: A Venn Diagram Relating the AV Paradigm to its Subcases

These potentially asymmetric ex-post utility functions are common knowledge, but $S_{i}$ is privately known and, in the model, represents private information the bidders might have concerning the realization of $V$. Note that, before bidding occurs, $V$ is unknown to the bidders but after the outcome of the auction is determined, its realized value $v$ is often assumed to be observable to the winner. In general, the ex-ante utility for bidder $i$ is a function of the signals of all bidders, as each signal provides useful information concerning the realized value of $V$ :

$$
U_{i}=U_{i}(\mathbf{S})
$$

A key distinguishing feature of CV models is the presence of the winner's curse. The winner's curse occurs in an equilibrium with monotonic strategies as bidders realize that winning delivers the unwelcome news that their signal was the most optimistic estimate of the object's worth. Wilson (1977) showed that rational bidders will respond by conditioning on the event that their signal is the highest, which imposes a downward pressure on their bidding strategies. The winner's curse is also known to intensify as $\mathcal{N}$ increases, which amplifies the tendency to shade bids. The result is that equilibrium bids in $\mathrm{CV}$ auctions need not be monotonic with respect to the number of bidders and expected revenue may potentially decrease with higher levels of participation. This phenomenon is empirically important because it places restrictions on equilibrium bidding when the number of bidders varies across observed auctions. It has become the basis for many empirical tests of what is known as the symmetric pure common values (PCV) model against independent private values models (discussed below). The PCV model imposes one additional restriction on the common values structure we've outlined. Specifically,

$$
u_{i}\left(S_{i}, V\right)=u(V), \quad i=1, \ldots, \mathcal{N}
$$


i.e., all bidders' ex-post appraisals of the item's true value are identical. The PCV model is the most commonly studied model within the $\mathrm{CV}$ paradigm in the empirical literature $3^{3}$ Note, too, as shown in Figure 1, the PCV model is also a special case of the CIPI paradigm which we discuss below.

\subsection{Affiliated Private Values}

The APV model is a special case of the AV model where ex-post utility of having won the object is fully characterized by the private signal $S_{i}$. That is, ex-post utility is given by

$$
u_{i}\left(s_{i}, v\right)=u_{i}\left(s_{i}\right)
$$

Hence, all bidders value the object differently, ex post. In APV settings, $V$ is a signal that conveys information on the values of one's opponents. The APV paradigm is often an attractive framework for empirical research because of the generality that it affords as it nests any private values model, including the popular independent private values paradigm (see below) as a special case.

Pinkse and Tan (2005) showed that, in any information structure with affiliated private information, there exists a strategic affiliation effect which causes bidders to behave as if they were subject to the winners curse, as in a CV model (see discussion in Section 7.6.1 below). The affiliation effect has important implications for efforts to distinguish between common- and private-values environments. It implies that APV models are not distinguishable from CV models based solely on observations that might appear to be generated by the winner's curse. Given the strategic similarities in the APV and CV paradigms, the APV paradigm can naturally produce bidding patterns that arise in either of the two polar extremes of independent private values or pure common values, as well as models that lie in between. Of course, a downside of this generality is that identification of the structural elements of the model is often difficult to achieve - something we later discuss.

Two special cases of the APV paradigm deserve particular attention: the conditionally independent private values (CIPV) model and the independent private values (IPV) model. The CIPV model is a special case of APV in which the $S_{i}$ s are independently distributed, conditional on unknown realizations of $V$, which are assumed to be affiliated with the $S_{i}$ s. Here, the random variable $V$ serves to introduce correlation into the otherwise unrelated private signals of the bidders. When $V$ is irrelevant to bidders' decisions and the joint density of signals is a product of independent marginal densities,

$$
f_{\mathbf{S}}(\mathbf{s})=\prod_{i=1}^{\mathcal{N}} f_{S_{i}}\left(s_{i}\right),
$$

then the IPV model applies. In this case, the inequality that defines log-supermodularity of $f$

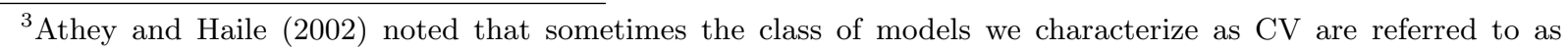
"interdependent values," with the term "common values" being reserved specifically for the PCV model. While both taxonomies are used in the literature, Athey and Haile chose to follow the one which emphasizes the distinction between statistical properties (independence, affiliation, etc.) of bidders' private information and the economic nature (private vs. common value) of this information. We adopt the same convention in this survey.
} 
holds with equality. Note that the IPV model is a special case of CIPV model in which $V$ does not create strict correlation among private signals. The nesting relationship, IPV $\subset \mathrm{CIPV} \subset \mathrm{APV}$, is depicted in Figure 1.

While the IPV model is fairly restrictive, it is the most widely adopted model by applied researchers. IPV models have some special characteristics which are important empirically. Namely, when bidders are symmetric and risk-neutral, and when a single object is for sale, the IPV paradigm exhibits a special characteristic called the revenue equivalence property (REP): for any auction format in which the highest bidder wins the auction with certainty, any symmetric and increasing equilibrium where bidders with signal $\underline{s}$ have expected utility of zero renders the same expected revenue to the seller; see Riley and Samuelson (1981) as well as Myerson (1981). Hence, choice of auction format becomes irrelevant, although only under this set of fairly restrictive conditions. Therefore, observations of significant revenue dominance among different auction formats can be used to reject the symmetric IPV model with risk neutral bidders. Milgrom and Weber (1982) showed that for the general AV framework with risk-neutral bidders, the four traditional auction formats can be revenue ranked as follows: expected revenue from English auctions weakly exceeds expected revenue from Vickrey auctions, which weakly exceeds expected revenue from first-price, sealed-bid or Dutch auctions. Maskin and Riley (1984) showed that when bidders' preferences for the object involve risk-aversion, expected revenue from a first-price, sealed-bid auction dominates that of a Vickrey auction. See Chapter 4 of Krishna (2002) for more examples of REP failures.

Finally, it should be mentioned that for every private-value model there exists an analogous private-cost procurement auction where bidders are not consumers, but firms bidding for a contract to provide a service, and where the low bidder is dubbed the winner. The two settings are very similar, with most qualitative intuition on information environment and equilibrium behavior carrying over seamlessly. To avoid confusion we shall frame our discussion in terms of the private value setting whenever possible, but this is without loss of generality.

\subsection{Conditionally Independent Private Information}

The CIPI model is a special case of the AV framework in which a particular form of affiliation among the random variables $(\mathbf{S}, V)$ is assumed. Specifically, signals $S_{i}$ are distributed independently, conditional on realizations of $V$. Thus, the joint density $f_{(\mathbf{S}, V)}$, assumed to be affiliated, is given by

$$
f_{(\mathbf{S}, V)}\left(s_{1}, \ldots, s_{\mathcal{N}}, v\right)=f_{V}(v) \prod_{i=1}^{\mathcal{N}} f_{S_{i} \mid V}\left(s_{i} \mid v\right) .
$$

The model studied by Wilson (1977) actually falls into this category, and Milgrom and Weber (1982) generalized Wilson's framework to allow for a broader variety of affiliation in the random variables $(\mathbf{S}, V)$. To the best of our knowledge, Li, Perrigne, and Vuong (2000) were the first to introduce this information structure into empirical work. They considered only the case where signals are both independent and identically distributed, conditional on $V$, because such an assumption guarantees that $(\mathbf{S}, V)$ are affiliated. One can certainly imagine an asymmetric CIPI structure, but additional 
assumptions on the marginal densities of private information may be necessary to preserve affiliation of the joint density.

The structure imposed in CIPI models allows them to nest as special cases both the CIPV, when $u_{i}\left(s_{i}, v\right)=u_{i}\left(s_{i}\right)$, and the PCV, when $u_{i}\left(s_{i}, v\right)=u(v)$. This also means that this special family of models overlaps both of the two traditional divisions of the AV paradigm. In fact, Li et al. (2000) (in their footnote 3) showed that by de Finetti's theorem any symmetric APV model is nested within the CIPI family. For this reason the CIPI model is an attractive model for empirical workespecially when bidders are symmetric. Li et al. (2000) (in their Proposition 1) also showed that CIPI inherits a common characteristic from its AV parent: just as any AV model is equivalent to some APV model, any CIPI model is observationally equivalent to some CIPV model. Therefore, a difficulty of adopting a CIPI assumption is that additional restrictions are needed in order to obtain identification. One strategy employed by Li et al. (2000) as well as Hong and Shum (2002) is to assume that private signals can be decomposed in the following way:

$$
S_{i}=\eta_{i} V
$$

where the $\eta_{i}$ s and $V$ are mutually independent and the $\eta_{i}$ s are identically distributed.

\subsection{Additional Comments on the Informational Paradigms}

Before moving on we introduce a few final pieces of notation: let $t \in\{1,2, \ldots, T\}$ index a set of auctions for which the econometrician is able to observe relevant data. The set of observables gleaned from each individual auction can vary along several dimensions. One study may incorporate data on only the winning bid, denoted $W_{t}$, from each auction, whereas another may incorporate data on all bids, $\left\{b_{1 t}, b_{2 t}, \ldots, b_{\mathcal{N} t}\right\}$ into estimation. Moreover, some papers assume the number of bidders is known and some do not. Finally, it can also be useful at times to incorporate sets of auctionspecific covariates, denoted by a vector $\mathbf{Z}-e . g$., the condition and characteristics of the object for

sale - into estimation, though such information is not always available or even feasible to include in estimation. Naturally, different sets of observables will lead to identification (or non-identification) of different aspects of an auction model, and will play a significant role in the discussion below. Generally speaking, as model complexity increases, either richer sets of observables or more stringent assumptions (or both) are required for model identification.

As for a brief description of equilibrium bidding in auctions, it can become quite complex in general settings, but it is useful to build intuition from the simple case of IPV with symmetric, riskneutral bidders, where bidding behavior under standard auction formats is simple and intuitive. For second-price auctions (including both English and Vickrey), the dominant-strategy equilibrium is to bid one's private value, or $\beta\left(s_{i}\right)=s_{i}$ for all bidders $i$. In first-price auctions, equilibrium bidding is given by the following relation:

$$
\beta\left(s_{i}\right)=\int_{\underline{s}}^{s_{i}} q \frac{(\mathcal{N}-1) F_{S}(q)^{\mathcal{N}-2} f_{S}(q)}{F_{S}\left(s_{i}\right)^{\mathcal{N}-1}} d q .
$$


Although bidding is generally nonlinear in this case and closed-form solutions rarely exist, the above expression has a very simple interpretation: each bidder selects as her bid the expected highest competing private value, conditional on all competitors' values being less than her own. This interpretation no longer applies to equilibrium bidding in more general private value settings, but it is still useful to think of them in relation to the simple benchmark model. For example, bidders will bid strictly higher for a given $s$ if they are risk averse, but if there is positive affiliation in their private values then they will bid strictly less. On the other hand, all private-value second-price auctions for a single object have the same dominant-strategy equilibrium.

It is worth noting that our above discussion of the theoretical models allows for bidders to have heterogeneous utility functions. In econometric practice, however, this level of generality creates substantial difficulties in model identification and would require bidder-specific information to be extracted from a given data set, something which is often difficult to achieve. Throughout this survey, we focus on the case in which all bidders share the same utility function because no paper that we are aware of considers the heterogeneous utility case. Moreover, most researchers consider estimation of the distribution(s) of latent characteristics for risk-neutral bidders. We discuss work that relaxes this assumption in our special-topics section concerning bidders who are risk averse.

Having outlined the different information structures in auctions, we now turn our attention toward research that has developed empirical methods within each of the respective paradigms. Hereafter, we organize the literature according to the information structure that is studied in a given work. In order to give a rough sense of how the literature has evolved, for the most part we attempt to organize papers within each information structure chronologically, according to publication date or latest-version date for unpublished works. 4 Exceptions to this policy occur only in cases where expositional flow is enhanced by chronological digressions, such as when followup papers are produced which have strong connections to earlier works (e.g., Donald and Paarsch (1993), Donald and Paarsch (1996), and Donald and Paarsch (2002)). We begin by discussing the most specialized paradigm described above - the IPV setting, in the next section. We follow this by discussing the CV paradigm in Section 4 as well as the APV and CIPI models in Sections 5 and 6 , respectively. We postpone discussions concerning work that focus on the special topics we consider until Section 7.

\section{Independent Private Values Paradigm}

We begin with the IPV paradigm for two reasons. First of all, estimation in this case tends to be the most straightforward, relative to the other information structures. A second reason is that the IPV paradigm has often served as a laboratory for developing new estimation methods which have then been adapted to handle more complex circumstances. Two prominent examples of this are IPV estimation techniques developed by Laffont, Ossard, and Vuong (1995) and Guerre,

${ }^{4}$ The experienced reader will realize that this need not perfectly reflect the actual chronology, as publication ordering need not correspond to the order in which the ideas actually appeared as working papers for the first time. Thus, this organization is intended only to convey a rough sense of evolution. 
Perrigne, and Vuong (2000) that have been extended to allow for estimation in more general APV models, and even in models of auctions for multiple objects. In this section, we only cover papers which have explicitly assumed IPV when specifying the structural model of bidding. There are other papers not covered here, which have estimated IPV models as a special case of an estimation procedure designed to handle more general informational paradigms. These will be covered in later sections.

Paarsch (1992) is widely considered to be one of the founding works of the structural empirical auctions literature. In this pioneering work, Paarsch employed parametric methods to study procurement auctions for tree-planting contracts in British Columbia, Canada. In his procurement model, signals took the form of privately-known production costs among firms competing for contracts. The contract was awarded to the firm submitting the lowest bid. Paarsch derived expressions for the data generating process of winning bids within three simple distributional specifications that admit explicit solutions: Pareto, Weibull and Exponential. From these derivations he was able to estimate the model by deriving explicit formulas for several objects tied to the distribution of winning bids - which is itself a function of the private-value distribution - under these three specifications. The derivations then facilitate two alternative estimation procedures. First, maximum likelihood (ML) may be applied to estimate model parameters using the derived density of winning bids to construct the likelihood function. Alternatively, a nonlinear least squares (NLS) approach, based on

$$
w_{t}^{r}=R_{r}(\boldsymbol{\theta})+e_{t}
$$

may be applied, where $w_{t}$ is the observed winning bid for the $t^{\text {th }}$ auction in the sample, $\boldsymbol{\theta}$ is a vector of parameters, $R_{r}$ is a function representing the $r^{\text {th }}$ raw moment of the distribution of winning bids and $e_{t}$ is an independent random variable with zero mean.

Donald and Paarsch (1993) recognized that standard ML estimation is problematic in first-price auction models, as the support of the distribution of bids depends on parameters of the valuation distribution. Such problems obtain in other econometric models whenever the support of a random variable depends on some of the parameters of its density function; e.g., for uniform and Pareto random variables. In the first-price auction setting, the researcher observes one random variable (bids) but is interested in estimating parameters of another (the latent valuation distribution). Using a change of random variables to map bids to valuations implies the Jacobian of the bidding function appears in the likelihood of interest, making computation difficult. More importantly, the finite upper bound of support of the bid distribution depends on the parameters of the valuation distribution (even if its support is unbounded). In this event, a regularity condition required to apply the standard (asymptotically normal) ML estimator does not hold which makes it difficult to derive the estimator's asymptotic distribution or even to show consistency; see, for example, Amemiya (1985).

Rather than abandon maximum likelihood, the authors suggested a modification based on order statistics which they termed piecewise pseudo-maximum likelihood (PPML) estimation. The estimation scheme proceeds as follows: partition the observed data by the number of rivals at each 
auction (hence the piecewise part) and take the most extreme winning bid from that subsample. One parameter of the valuation distribution can be recovered from each subsample by requiring that, for a given parameter vector, the implied bid support must contain even the most extreme observed bids. This condition allows one parameter to be expressed as a function of the others which, when written this way, can be substituted into the (now) pseudo likelihood function which can be maximized with respect to the other parameters. Framing the objective as a constrained optimization problem allowed Donald and Paarsch to link distribution theory of order statistics with how the estimator is computed in practice to establish its asymptotic theory. In order to demonstrate the properties of the estimator, Donald and Paarsch derived a set of conditions under which it is consistent and asymptotically normal. It turns out that the estimator is also well-behaved in small samples, as shown by a Monte Carlo study.

Donald and Paarsch (1996) followed up on this initial research by discussing identification of the four common auction formats using parametric methods. They extended the work of Donald and Paarsch (1993) to fully solve the support-related problem, allowing for discrete covariates 5 Donald and Paarsch (2002) proposed a new extreme-order, generalized methods-of-moments (MOM) estimator which retains some important properties of ML estimators but has the advantage of being easier to compute and is superconsistent having exponential limiting distributions. By means of Monte Carlo experiments, they demonstrated that their estimator performs well in small samples. Finally, they used it to re-evaluate the tree-planting data from Paarsch (1992), finding evidence consistent with his original findings.

Laffont et al. (1995) proposed a new estimation method called simulated nonlinear least squares (SNLS), which addressed a criticism of parametric methods such as ML, NLS, and MOM that arises when the inverse-bid function has no closed-form solution: they are computationally burdensome. This difficulty occurs due to the need to solve integrals numerically for each trial value of the parameter vector during optimization of the empirical criterion function. Laffont et al. proposed replacing direct computation with a simulation method based on the revenue equivalence principle. Suppose the researcher observes a sample of winning bids, $\left\{w_{t}\right\}_{t=1}^{T}$, where, for simplicity sake, the number of bidders is fixed at $\mathcal{N}$ in each auction. She wishes to minimize the average quadratic distance between the members of the sample and the theoretical average winning bid $m(\boldsymbol{\theta})(\boldsymbol{\theta}$ contains the parameters of the private value distribution), but the trouble is that $m(\boldsymbol{\theta})$ has no closed-form solution. However, since revenues in a first-price auction within the risk-neutral IPV paradigm are the same, on average, as in a Vickrey auction, it follows that the expected winning bid in a first-price auction is the same as the expected second highest valuation among $\mathcal{N}$ bidders (i.e., the sale price in a Vickrey auction).

Thus, for given values of the parameters, a replacement for $m(\boldsymbol{\theta})$ may be simulated by drawing

\footnotetext{
5 Chernozhukov and Hong (2004) developed Bayesian likelihood-based estimation and inference methods in econometric models with general regressors for which there are jumps in the conditional density and discontinuous likelihood functions. They noted that the models considered by Donald and Paarsch are an example in which the conditional density of winning bids jumps from zero to a positive value. Depending on one's notion of loss functions, Bayesian methods can often be more efficient.
} 
$K$ samples of size $\mathcal{N}$ from the value distribution $f_{S}(s ; \boldsymbol{\theta})$ and computing the average of the secondhighest valuation over all the simulated samples, $\bar{X}_{K}(\boldsymbol{\theta})$. Once again, by the REP, it follows that $\mathrm{E}\left[\bar{X}_{K}(\boldsymbol{\theta})\right]=m(\boldsymbol{\theta})$. A natural estimator then becomes

$$
\arg \min _{\boldsymbol{\theta}}\left\{\frac{1}{T} \sum_{t=1}^{T}\left[w_{t}-\bar{X}_{K}(\boldsymbol{\theta})\right]^{2}\right\} .
$$

One remaining problem is that the estimator implied by equation (1) is inconsistent for any finite $K$, for reasons similar intuitively to errors-in-variables bias in standard regression models. Luckily though, in this case, a relatively straightforward (but tedious) expression for the bias can be derived which depends only on the variance of the simulation estimator $\bar{X}_{K}(\boldsymbol{\theta})$. Once this correction term is added into equation (1) (see Laffont et al. for further details), a consistent and computationally-inexpensive estimator results. Although this is the basic idea behind the approach, Laffont et al. actually put forward a slightly more complicated simulation scheme involving importance sampling, which ensures differentiability of the empirical objective function. They showed that their estimator is consistent and asymptotically normal, and they derived the limiting form of the variance-covariance matrix. Moreover, they also outlined how estimation may be adapted to deal with unobserved heterogeneity or imperfect knowledge of the total number of bidders - two issues we return to in the last section of this survey. They also illustrated the procedure by applying it to a sample of descending auctions for French agricultural products (eggplants).

Elyakime, Laffont, Loisel, and Vuong (1997) studied first-price, sealed-bid auctions in which the reservation price is unknown to bidders - this has become known as a "secret reserve price." Similarly as in auction theory, the reservation price is modeled as a random variable from the bidders' perspective. The authors allowed for the possibility of a Nash-bargaining process under complete information between the seller and the highest bidder if the auction does not result in a sale. Equilibrium strategies involve rational bidders taking into account the possibility of secondround negotiations when formulating their first-round bids. The authors derive these strategies for both bidders and sellers and showed that the distributions characterizing the buyers' and sellers' private values are identified nonparameterically from observations on bids and reservation prices. Elyakime et al. proposed estimating these structural elements of the model parametrically by joint NLS while using a backward differentiation formula to solve for the equilibrium biding strategy for a given guess of the parameter vector. Specifically, the authors assumed the bidders' and sellers' valuation distributions followed Weibull laws. The parameters of these were chosen to minimize a NLS objective which weighted equally the squared differences between the first moments of the observed bids, reservation prices, and transaction prices (for items not sold during the first-round auction process) and their respective expectations given the parametric structure. They performed this procedure on a sample of French timber auctions and found that this strategic model achieves a better fit than one with myopic bidders who ignore the possibility of second-round negotiations.

A common thread in all of the above papers is that they all employed parametric methods to recover the structural elements of the model. Such methods have been criticized on two main grounds, 
one statistical and the other computational. The former criticism is that they require explicit distributional assumptions on bidder heterogeneity and, thus, may be subject to misspecification errors. The latter is that parametric estimators are expensive computationally and/or difficult to implement. Guerre et al. (2000, GPV) addressed both of these issues by proposing a fully nonparametric two-stage estimation strategy which eliminates the need of iteratively optimizing an empirical criterion function. Indeed, while the importance of the misspecification criticism has been debated, the computational advantage truly makes the work of GPV a landmark accomplishment - one need only reflect on the fact that only a few parametric specifications of private signals render explicit solutions for equilibrium bidding, to see how important this new advance was to empirical auction work.

The main innovation of GPV stems from the following observation: in equilibrium within a first-price auction it holds that (letting $B=\beta(S)$ denote an equilibrium bid)

$$
F_{B}(b)=\operatorname{Pr}(B \leq b)=\operatorname{Pr}\left(S \leq \beta^{-1}(b)\right)=F_{S}\left(\beta^{-1}(b)\right)=F_{S}(s)
$$

thus,

$$
f_{B}(b)=\frac{f_{S}(s)}{\beta^{\prime}(s)} .
$$

With this transformation of random variables in mind, the standard differential equation for equilibrium bidding within the IPV paradigm with symmetric, risk-neutral bidders,

$$
1=(s-\beta(s))(\mathcal{N}-1) \frac{f_{S}(s)}{F_{S}(s)} \frac{1}{\beta^{\prime}(s)},
$$

may be transformed into the following equation:

$$
s=\xi\left(b, F_{B}, \mathcal{N}\right) \equiv b+\frac{1}{(\mathcal{N}-1)} \frac{F_{B}(b)}{f_{B}(b)} .
$$

In other words, just as equilibrium bidding can be expressed as a monotone function of the (unobserved) private signals and their unknown distribution, private signals may alternatively be expressed as a monotone function of observed equilibrium bids and their (directly estimable) distribution. Thus, in the first stage proposed by GPV, the econometrician directly recovers an estimate $\widehat{F}_{B}(b)$ of the bid distribution and its density nonparametrically. At this point a sample of pseudoprivate values can be constructed using the above inverse-bidding function $\xi(\cdot)$. These estimates are dubbed "pseudo-values" rather than values because they are recovered using an estimate of the density of bids, rather than the actual density itself. In the second stage, the econometrician may use this sample of pseudo-values to nonparametrically recover the density and distribution of private values.

One final comment on the statistical efficiency of their strategy is worth mentioning before we move on. It is well-known that kernel density estimators display a considerable amount of downward bias near the boundaries of the support, a phenomenon known as the boundary effect. 
Simply stated, a kernel density estimator at a given point averages kernel function values within a neighborhood of that point, but for points within a bandwidth length of the extremes of the support no data can exist on the other side of the boundary. Thus the kernel density estimator naturally penalizes the estimate downward within that neighborhood. Since boundary effects would cause problems for the second stage of estimation, given that pseudo-values are based on such estimates, GPV proposed a solution to this problem by sample trimming, or discarding first-stage pseudo-value estimates based on kernel density estimates in the problematic region of the support. They showed that by performing this intermediate step, the estimated distribution $\hat{F}_{S}(s)$ achieves the optimal rate of convergence to $F_{S}(s)$. Of course, the optimal rate of convergence comes at a price - throwing out some of the data near the extremes of the sample of pseudo-private values gives rise to a larger region of bias at the extremes of the support of $S$ for finite samples.

The method developed by GPV has since been extended to several more general frameworks outside the symmetric IPV paradigm. GPV is the most widely-cited paper in the structural econometric literature concerning auctions and often serves as a benchmark which current research continues to build off or which new methods are compared to, as will be clear from the discussions that follow.

In more recent work, Hickman and Hubbard (2012) proposed a modification to GPV which circumvents certain complications arising due to sample trimming. One is straightforward: sample trimming implies a loss of data. However, two additional complications are more nuanced; for simplicity, we shall concentrate our discussion of these on the upper bound of the support of private values. First, sample trimming precludes the possibility of nonparametric estimation or inference within the following set: $\left(\hat{\xi}\left(B_{\max }-h_{B}\right), \bar{s}\right]$, where $\hat{\xi}$ once again denotes the estimated inverse bidding function, $B_{\max }$ denotes the highest observed bid, and $h_{B}$ denotes the first-stage bandwidth. Moreover, because the sample of pseudo values is also kernel smoothed in the second stage, there is an additional, adjacent region where $\hat{f}_{S}$ will exhibit excessive downward bias due to boundary effects. Thus, for a given pair of first- and second-stage bandwidths $\left(h_{B}, h_{S}\right)$, sample trimming causes inference to be either problematic or impossible within the following set:

$$
\left(\xi\left(\bar{b}-h_{B}\right)-h_{S}, \bar{s}\right]
$$

where $\bar{b}$ denotes the upper bound on the bid support, and with a similarly-defined interval at the lower boundary as well. Let $\Upsilon$ denote the union of the upper and lower problematic intervals. Hickman and Hubbard noted a final complication from sample trimming: it invalidates standard bandwidth selection criteria (derived with single-stage estimators in mind), because optimal bandwidth selection in the first stage involves a trade-off between the size of the set $\Upsilon$ and the statistical properties of $\hat{f}_{S}$ on the interior. Hickman and Hubbard proposed a solution to these problems by replacing sample trimming with recently-developed statistical techniques for boundary correction which allow kernel density estimators to have uniform bias and variance on the closure of the support of the distribution. In Monte Carlo studies involving various private value distributions, they demonstrated the finite-sample gains to be had from replacing the standard kernel estimator with 
sample trimming by a boundary-corrected kernel estimator in each stage of GPV.

A major contribution to the literature, once GPV had shown that symmetric first-price auctions could be identified (and estimated) nonparametrically, was establishing a set of results concerning nonparametric identification in more general settings. Athey and Haile (2002) did so within various different auction formats and information structures and also explored questions of what minimal samples of observables would allow for nonparametric identification in the various combinations of the former and the latter. Athey and Haile argued that these identification results provided valuable insights concerning use of both nonparametric and parametric techniques. On the one hand, they indicate which models may be estimated without resorting to distributional assumptions. On the other hand, they also better inform our use of parametric assumptions. In the authors' own words,

When a model is nonparametrically identified, one can view a parametric specification as a parsimonious approximation rather than a maintained hypothesis...conversely, nonidentification results can both demonstrate why the data fail to enable inferences of certain types and suggest the range of models that could generate the same observablessomething valuable for policy-makers interpreting results obtained with strong identifying assumptions. (Athey and Haile (2002, pg. 2109))

Not surprisingly, in the simple case of IPV, identification is a very simple matter. In the English and Vickrey action formats with symmetric bidders, identification requires only observations of the transaction price. When bidders are asymmetric, information on the identity of the bidder is also required. Athey and Haile derived an extensive set of identification results within more general information structures, to which we will refer later on in the survey. Finally, in addition to identification, they also studied the issue of testability, that is, whether equilibrium behavior in a given model involves refutable restrictions on the distribution of observables in a variety of settings.

The primary focus of our discussion thus far, as well as in going forward, concerned first-price auctions. One reason for this is, regardless of whether bidders are symmetric or not, the weakly dominant strategy at a second-price auction is for players to bid their valuations. As such, the structural exercise simply requires mapping order statistics to their parent distribution (for details, see Chapter 3 of Paarsch and Hong (2006)). However, Haile and Tamer (2003) addressed what they argued is an important discrepancy between standard theoretical models of oral, ascending auctions and those observed in practice. The standard theoretical model, known as the MilgromWeber "clock model," is one in which the sale price rises continuously as bidders reaffirm their willingness to pay, dropping out precisely once the price exceeds their private value. However, they argued that in practice sale prices adjust discretely by increments of varying sizes, as competitors call out bids at will. This may result in sale prices being not perfectly equated with the secondhighest private value, making precise recovery of the value distribution problematic.

Rather than recover an exact estimate, Haile and Tamer (2003) instead established partial identification of an incomplete model of bidding based on two weak assumptions that are required by (but not sufficient for) Nash equilibrium bidding: first, bidders never bid more than their private value and, second, bidders never allow an opponent to win at a price they would have been willing 
to beat. Using the first assumption, they obtained an upper bound on the distribution of private values and similarly, the second assumption provided a lower bound. Moreover, they showed how the resulting set estimates could be used to bound optimal reserve prices. Finally, they used Monte Carlo experiments and an example with U.S. timber auction data to demonstrate that meaningful, tight bounds may be obtained. Furthermore, they showed that the assumption of continuous price adjustments can lead to estimates that lie outside of their bounds.

Li and Perrigne (2003) analyzed first-price auctions with secret reserve prices. Like Elyakime et al. (1997), they modeled the reserve price as a random variable drawn from a distribution which is common knowledge to bidders. Thus, the structural elements to be estimated include both the bidders' private value distribution and that of the seller. Given this extra latent variable, the differential equation characterizing equilibrium bidding is different from the canonical case where the reserve price is known. The authors derived this condition and extended GPV by reworking their original two-step estimation procedure with this more complicated differential equation in mind. Li and Perrigne then applied their proposed estimator to a sample of French timber auctions in which reserve prices were actually chosen at random and withheld from bidders' knowledge prior to bidding. After estimation, they concluded that implementation of the optimal reservation price would render higher revenues to the French government.

Flambard and Perrigne (2006) extended the GPV method to the case where bidders' private values are asymmetrically distributed. This method is particularly advantageous in the presence of asymmetric bidders because of the intractability of equilibria in such auctions. Specifically, with heterogeneous bidders a system of differential equations arises which does not satisfy a Lipschitz condition needed for integration. Solving these models requires advanced numerical methods to solve the system of equations given the value of parameters at each iteration (say, within a nestedfixed point procedure). As such, computational difficulties are exacerbated significantly in such settings - see Hubbard and Paarsch (2011). To circumvent this, Flambard and Perrigne transform this system of equations in a way similar to GPV for the symmetric (single equation) case to solve for private values in terms of bids and the bid distributions. Once the first stage of estimation is complete, the trimming phase and second stage remain basically unchanged. To illustrate their approach, Flambard and Perrigne applied their estimator to auctions for snow removal contracts in Montréal, Canada in which observable differences between bidders are correlated with bid levels.

While Flambard and Perrigne (2006) extended GPV to an asymmetric environment, identification and estimation of their model require knowledge of which bidders submitted the observed bids. Lamy (forthcoming) extended GPV to first-price and second-price auctions in which bidders' identities, for whatever reason, are not observed, but for which the econometrician might want to allow for bidders to be asymmetric. He showed a model with anonymous bids is identified up to a permutation of the potential bidders' identities and his identification argument suggests an estimation routine which adapts that of GPV. Given a sample of auctions, a researcher can construct an estimate of the distribution of the order statistics of the bids. In an IPV setting, the distribution of each bidder's bids at a given bid can be expressed as the roots of a polynomial whose coefficients 
are a linear combination of these estimated distributions of order statistics. He proposed recovering a pseudo private value for every possible realization of the identity of the bidder and estimating a vector of pseudo probabilities mapping each observed bid to each potential identity, after which the distributions of valuations are estimated. Lamy's multi-step adaptation of the GPV algorithm achieves the optimal rate of convergence.

\section{Common Values and Pure Common Values}

As in the previous section, here we only cover papers which have explicitly assumed CV or PCV when specifying the structural model of bidding. As mentioned previously, it is well-known that in CV auctions, the winner of an auction is faced with the bad news that she had the most optimistic signal of any competitors, giving rise to the winner's curse. As Wilson (1977) showed, rational bidders will compensate for the winner's curse by conditioning their bid on being the most optimistic.

Thiel (1988) was perhaps the first to directly incorporate a derived expression for equilibrium competitive bidding into an econometric auction model with pure common values. By assuming that the private signal distribution is a member of the location-scale family of distributions, he was able to derive a bidding function which is linear in its parameters. From this starting point, he constructed an empirical specification of bidding that could be estimated by least squares. Estimation of the magnitude of the winner's curse then translated into a simple hypothesis test on the regression parameters. Thiel (1988) applied his empirical model to a sample of 150 auctions for highway contracts across 33 various U.S. states.

McAfee and Vincent (1992) developed a simple test that is asymptotically distribution free for whether the reserve price set by the seller is inefficiently low in first-price, CV auctions. Roughly, they showed (Theorem 2) that the reserve price is too low if the seller's expected value of the object exceeds the expected value of the object, conditional on the highest bid being the reserve price. Note, however, that this result provides only a necessary condition for reserve price optimality, and not a means of producing a precise estimate of the optimum. Nevertheless, McAfee and Vincent showed that their test can have practical significance by applying it to a sample of outer continental shelf (OCS) wildcat auctions in which they found that the reserve price set by the U.S. Department of the Interior (DOI) was far too low.

As in the IPV case, Paarsch (1992) was the first to derive explicitly the data generating process of winning bids within the CV information structure. He did so for two specifications of the distribution of private signals - Weibull and normal - and proposed estimating these distributions via NLS or ML. He also suggested a method of testing between common and private values, which we later discuss. After Paarsch (1992), work on estimation in the CV paradigm has since been sparse, and results proved by Athey and Haile (2002) shed some light on why this might be the case. Specifically, identification within the CV paradigm is considerably more difficult than under

private values. For example, even in the second-price, sealed-bid case, the simplest auction format 
for estimation purposes, identification of the private signal distribution requires observation of all bids, rather than just the winning bid. Even then, the joint distribution of private signals and the common value is unidentified. Naturally, identification of more complex auction formats such as the open, Dutch, or first-price, sealed-bid become even more difficult.

Some authors have used actual observations of the ex-post realization of the common value to overcome these difficulties. For example, Hendricks et al. (2003) analyzed data on auctions for mineral extraction leases on the Gulf of Mexico, in which bidders are unsure beforehand about the mineral content of a particular tract of ocean floor. These OCS auctions are special in that reliable estimates were available for the ex-post value of each tract. The authors were primarily interested in leveraging the realized value of the auction to test an hypothesis concerning bidder rationality. When the ex-post value of the object is observable, it is possible to test directly how well bidders compensated for the possibility of the winner's curse.

Hendricks et al. (2003) developed three tests for bidder rationality using these insights. The first test simply considered whether bidder rents were, on average, positive. The second test extended this to determine whether firms earn positive rents, conditional on submitting a winning bidthat is, profit margins should also be positive on average. Both of these tests were consistent with rationality, illustrating the remarkable power of the market to aggregate private information. However, the authors noted that both tests involved aggregation and argued that additional and, perhaps, stronger evidence could be found through a test on the individual level. Thus, the authors developed a test of individual rationality based on two observations. First, given the presence of the winner's curse, bidders should bid less than their expected tract value. It follows then that a rational bidder will submit a bid that is lower than her expected value, conditional on the number of potential bidders and her private signal. We shall call this expectation $E_{1}$. Second, a rational bidder will anticipate that, given a win, she will have, on average, overestimated the object's worth. Hence, she must also submit a bid that is less than her expected value of the object, conditional on the number of potential bidders, her private signal, and conditional on having the highest bid. We shall call this expectation $E_{2}$. Rationality requires $E_{1} \geq E_{2}$. If the winner's curse is present and bidders are rational, then it must follow that the difference $E_{1}-E_{2}>0$ is also strictly increasing in $\mathcal{N}$, the number of potential bidders. Hendricks et al. (2003) proposed using this difference as an estimate of the winner's curse. Specifically, the authors computed estimates of the conditional expectations via a locally linear regression ${ }^{6}$ Inference is performed using a block-bootstrap procedure to compute a one-sided $95 \%$ confidence band for the estimate of the winner's curse 7 The OCS data again proved consistent with the rationality hypothesis.

Hong and Shum (2003) developed an econometric model of English auctions within the CV paradigm. They considered risk-neutral bidders with distinct, unknown values for the object which depend on their own private information as well as that of their opponents. Moreover, their model allows for bidders' private signals to be distributed asymmetrically. English auctions in the

\footnotetext{
${ }^{6}$ Details concerning this nonparametric estimation procedure are in Appendix B of Hendricks et al. (2003).

${ }^{7}$ For details, see Appendix C of Hendricks et al. (2003).
} 
CV paradigm are exceedingly rich models in a strategic sense, and pose a formidable challenge to empirical researchers due to the way in which bidders learn and adapt their strategies as the auction progresses. Hong and Shum applied theoretical insights due to Wilson (1998) to make econometric implementation of the model feasible. Wilson discovered that the inverse-bid functions are pointwise defined implicitly by a system of nonlinear equations, and that the existence of a monotonic equilibrium is equivalent to conditions which deliver an increasing solution to the system. This discovery created enough parsimony to facilitate econometric implementation, provided that the latent distributions allow for workable, closed-form expressions.

Hong and Shum (2003) adopted a parametric assumption-log-normal private signals - which allowed them to derive the equilibrium bidding function and the likelihood function for the model. Moreover, they showed that the support of private signals does not depend on model parameters, so the ML regularity conditions hold and standard asymptotic theory applies. However, as implementation of ML involves the computation of a difficult, high-dimensional integral, they suggested optimizing the likelihood function using simulation techniques. They also adopted an alternative approach, based on simulated moments. This second method is effectively an extension of the SNLS estimator of Laffont et al. (1995) to the current model, but to preserve differentiability of the SNLS objective function, Hong and Shum abandoned the importance sampler used by Laffont et al. (1995) in favor of another based on the independent, probit kernel-smoother of McFadden (1996). Finally, using Monte Carlo simulations, Hong and Shum performed a sensitivity analysis of both methods to check for effects of changes in the bandwidth and the number of simulations. They found that, while both procedures performed fairly well, the SNLS estimator appeared more stable.

There are two notable papers based off the idiosyncracies of PCV auctions administered electronically through the online auction website eBay. Bajari and Hortaçsu (2003) used data on collectible coin auctions in which the CV assumption is motivated by informational asymmetries between buyers and sellers, along with the existence of a highly liquid resale market and the presence of speculative collectors. Due to the presence of speculative bidders who presumably have expertise in appraising coins, the authors also modeled bidders' private signals as being distributed asymmetrically. Like Hong and Shum (2003), Bajari and Hortaçsu were confronted with the fact that dynamic CV auctions pose a considerable computational challenge to the empirical researcher. They employed two coping strategies to make estimation more plausible. First, following Roth and Ockenfels (2002), they used the fact that eBay auctions terminate at a specified point in time to simplify auction dynamics. They modeled time as passing continuously during an initial period in which bidders may drop out and re-enter at will, after which there is a final period when they may submit only one bid; at this point the game is equivalent to a static second-price auction. Their second coping strategy was to estimate the model using a simulation-based Bayesian approach. They specified a prior distribution for model parameters and applied Bayes' theorem to study the properties of the posterior. Then they simulated the posterior using Markov-chain Monte Carlo methods. They offered several arguments in favor of this method over classical estimation, including 
relative computational simplicity, valid inference with fewer assumptions, and findings by Hirano and Porter (2003) as well as Chernozhukov and Hong (2004) that Bayesian methods in parametric auction models may be asymptotically more efficient than frequentist methods in some relevant cases; see also footnote 5 of this survey.

Lewis (2011) used eBay data concerning automobile auctions. Here the CV assumption is motivated by information asymmetries between buyers and sellers giving rise to an adverse selection phenomenon à la Akerlof (1970). Lewis assumed bidders to be symmetric as he was primarily concerned with the way in which sellers overcome the adverse selection problem. He found that the amount of information supplied to buyers and the sale price were highly correlated. This is consistent with a model in which sellers' optimal policy isto report only information that reflects positively on the condition of the sale item. Lewis rendered his model dynamics more tractable by employing the same abstraction as described above in Bajari and Hortaçsu (2003). In his model, this implied that the game essentially unravels to a static Vickrey auction in which bids are based on private signals, the number of competitors, and the number of signals received from the seller. Lewis proposed a new pseudo-maximum likelihood estimation routine in which optimization is a mixture of weighted least squares and quasi-Newton algorithms. He argued that the advantage of his method over Hong and Shum (2002), which we discuss below, or Bajari and Hortaçsu (2003) is that it deals with the curse of dimensionality remarkably well, allowing him to include over thirty covariates in the model. He found that sellers can significantly affect the sale price by providing large amounts of information to buyers.

\section{Affiliated Private Values}

Athey and Haile (2002) provided better news concerning the APV paradigm relative to the CV framework, although working in this general model is still more difficult than its special IPV case. The minimal set of observables needed to deliver nonparametric identification in symmetric APV first-price auctions are the two highest bids, from which the joint distribution of the two highest private values is identified (Theorem 7 ). If the identity of the winner is also available, then in the asymmetric case, the bidding functions for all bidder types are identified (Lemma 1). However, if any bid is not observed in symmetric APV first-price auctions, then the joint distribution of all private values is unidentified (Corollary 1). Athey and Haile proposed that in such cases where parametric assumptions will be necessary to obtain identification, if the identity of the winner is known then bidding functions may be nonparametrically estimated and compared to the ones obtained parametrically as a specification test.

Athey and Haile (2002) also showed that similar difficulties arise in second-price auctions within the APV: if even a single bid is unobserved, then the joint distribution of bidder valuations is nonparametrically unidentified. This is particularly troublesome in the second-price setting as the bid submitted by the winner is often unobserved by the econometrician. Komarova (2011) studied

this issue further by developing partial identification results based on increasingly severe restrictions 
on the observables in models where bidders may be asymmetric. Take a given subset of bidders $\mathcal{J}$, Komarova showed how information on prices when all members of $\mathcal{J}$ lose the auction can be used to derive an upper bound on the joint distribution of valuations for bidders belonging to the subset $\mathcal{J}$. She also showed how one can use information on prices when a member of $\mathcal{J}$ wins to derive a lower bound for this joint distribution. Komarova demonstrated via a numerical exercise that, when a majority of bids are observed, bounds on the marginal private value distributions can be fairly informative if the bounds are tight.

Li, Perrigne, and Vuong (2002) extended the two-step estimation method of GPV to the case of symmetric, first-price auctions within the APV paradigm. Allowing for affiliation complicates the first step of estimation, as there is dependence among the observed bids. As such, the first-order conditions involve the ratio of the conditional bid distribution to its conditional density, which the authors showed can be estimated by the ratio of the (unconditional) joint bid distribution to its joint density. These latter terms can be estimated nonparametrically by computing a product of univariate kernels of the bid of interest and the maximum of that bidders' rivals' bids. The authors, in their Proposition 1, also highlighted a remarkable property of first-price auctions within the APV paradigm that produces a specification test. The result has to do with what is known in the literature as rationalizeability. A sample of observables is said to be rationalizeable by a model if there is an equilibrium of that model that induces the observed distribution over the observables. Li et al. showed that a sample of bids is rationalizeable by a symmetric APV model if and only if the joint distribution of bids is symmetric and affiliated and if the inverse-bidding function is strictly increasing. This result implies that not all distributions may be rationalized by an APV model, despite its characteristic generality. This proposition provides the basis for a formal test of the symmetric APV specification, characterizing all of the game-theoretic restrictions posed by the model on the distribution of observed bids. The test is convenient as well, as computation of the inverse-bidding function already required for recovery of pseudo-private values in the first stage of estimation. The second stage of the estimation follows that of GPV or, if the researcher is interested in the joint density of valuations, a product of univariate kernels can be estimated. The authors proposed modified bandwidths for use in the second stage and, like GPV, showed that their nonparametric estimator is uniformly consistent on arbitrary open subsets of the interior of the support of private values.

Campo, Perrigne, and Vuong (2003) extended Li et al. (2002) to handle asymmetrically distributed private values. A difficulty arises because of interdependence among the asymmetric bidders, which precludes a strict application of the method developed by Li et al. to each group separately. Specifically, each bidder evaluates the probability of winning the auction by considering the joint distribution of maximal rival bids from each type of player, conditional on those bids being less than her own bid. A complication is that the modified first-order condition associating observables with pseudo-values now depends on this conditional bid distribution as well as its total derivative. Thus, if there are $K$ bidder types, then the econometrician must effectively estimate a $K$-dimensional joint density, giving rise to the curse of dimensionality when conventional ker- 
nel smoothing techniques are employed. For this reason, Campo et al. confine their empirical implementation to auctions only involving two or fewer bidder types.

Another aspect of this paper that also deserves attention here. These stem from the fact that asymmetry in this model has a behavioral origin, and is not imposed exogenously. Campo et al. (2003) studied a set of OCS auctions during a time period in which the U.S. DOI allowed oil firms to form ad hoc cartels (at their own discretion) for the purpose of bidding and mineral extraction. This was an effort by the DOI to encourage participation by smaller firms. This type of asymmetry gives rise to a series of tests for the presence of bidder asymmetry and even of unobserved auctionspecific heterogeneity. As for the former, preliminary evidence for, or against, bidder asymmetry may be uncovered via a standard Z-test on the probability of joint bidders winning the auction. The idea here is that under the null hypothesis of symmetry, all bidder types should be just as likely to win a given auction. A second test for asymmetry comes after the second stage of estimation, when the marginal distributions of private values for each bidder type have been recovered. Campo et al. proposed applying a standard Kolmogorov-Smirnov test to these marginal densities to evaluate the null hypothesis of bidder symmetry.

Li, Perrigne, and Vuong (2003) developed a semiparametric estimation strategy of the optimal reservation price in first-price auctions when bidders are risk-neutral. In their model, the private values of bidders come from an affiliated joint distribution, meaning that each bidder's private value affects the conditional marginal densities of the other bidders' private values. The traditional expression for the optimal reservation price is a function of the distribution of private values; see, for example, Myerson (1981) as well as Riley and Samuelson (1981). However, within the APV paradigm they derived an expression characterizing the seller's expected revenue as a function of the bid distribution, from which it follows that a simple maximization routine gives rise to a natural estimator of the optimal reserve price. The authors studied OCS wildcat auction data for which they estimated the symmetric bidding function nonparametrically and incorporated this estimate into the seller's profit function. Li et al. then recovered the optimal reservation price via extremum estimation (also known as M-estimation). In light of Athey and Haile (2002), this was a significant breakthrough, as recovery of the optimal reserve price required identification of only the (inverse-) bidding function, which may be obtained even in some situations where the latent value distributions are nonparametrically unidentified.

Hubbard, Li, and Paarsch (forthcoming) proposed estimating first-price models in the symmetric APV paradigm semiparametrically. While the model is nonparametrically identified, assuming some additional structure allows the authors to address the curse of dimensionality (concerning the number of bidders at auction) and to "impose" affiliation in their estimation strategy, ensuring an equilibrium is satisfied by the first-order condition. Specifically, the authors employed Archimedean copulas to characterize the joint distribution of valuations. Copulas are functions that express the joint relationship between random variables as a function of their marginal distributions, and they allow the researcher to separate estimation of the marginal distributions from estimation of the joint distribution; see Nelsen (1999) for an introduction to copulas. This family of copulas is attractive as 
statisticians have identified conditions which arbitrary members must satisfy in order to guarantee affiliation holds.

Hubbard et al. estimated the marginal empirical distribution function and probability density function of bids nonparametrically, just like GPV. However, unlike GPV, they used the empirical distribution of bids in a (pseudo) maximum likelihood routine to estimate the copula parameter(s). These estimates allowed the authors to use the first-order condition characterizing equilibrium behavior in this model to recover pseudo values corresponding to each observed bid. They then suggested following GPV in the second step of their estimator. The authors showed their estimator of the joint density of private values achieves the optimal uniform rate of convergence for a two-step nonparametric estimator à la GPV - that is, the joint density of private values can be estimated at the same rate as the marginal density of private values. The copula parameterization allows for this asymptotic gain as the dimension of the joint density of private values is reduced to one which avoids the curse of dimensionality problem concerning the number of bidders and associated with the nonparametric approach of $\mathrm{Li}$ et al. (2002). The extra copula-related work in the first step appears to pay dividends in small samples: they used Monte Carlo experiments to show their estimator is on par with GPV when data are independent and that their estimator performs well on affiliated data, even if the researcher chooses the wrong copula. Furthermore, they found significant (and high) affiliation in cost signals using procurement data from a sample of Michigan Department of Transportation highway paving contracts.

\section{Conditionally Independent Private Information}

To our knowledge, there exist but two structural auctions papers within the CIPI paradigm, both of which were based off first-price auctions with symmetric risk-neutral bidders. However, together they represent a well-rounded sample of both parametric and nonparametric methods.

Li et al. (2000) introduced the CIPI paradigm into empirical research. They applied nonparametric estimation techniques to the two important special cases of CIPV and PCV. A significant obstacle they encountered was the fact that identification within the CIPI paradigm requires additional assumptions on the informational structure, due to the presence of the common component influencing all bidders' values alike. Thus, a researcher must recover not only the distribution over bidders' idiosyncratic signals, but also that of the common component. The authors accomplished this through a log-additive specification of firm costs, following Wilson (1998), where private information is decomposed as the product of the (unknown) common factor and a random idiosyncratic factor:

$$
S_{i}=V \eta_{i}
$$

Moreover, they assumed the $\eta_{i}$ 's and $V$ are mutually independent and that the $\eta_{i}$ 's are identically distributed with mean one. Using this assumption, along with a regularity condition from work in nonparametric deconvolution methods by Li and Vuong (1998), they showed that the CIPV model is nonparametrically identified from a sample of bids. Moreover, CIPV can rationalize a sample of 
bids if and only if the inverse-bid function is monotonic. As in Li et al. (2002), the power of this last result lies in the fact that their estimation procedure allows for verification of monotonicity, which in turn implies that the CIPV assumption is partially testable (subject, of course, to other sources of model misspecification).

On the other hand, in the PCV case, bidders use their private signals $S_{i}$ to estimate the common cost $V$, making identification difficult. As a step toward that direction, Li et al. $(2000)$ imposed the additional restriction

$$
\mathcal{E}\left[V \mid S(\mathcal{N}: \mathcal{N})=S(\mathcal{N}-1: \mathcal{N})=s_{i}\right]=C+D \log s_{i}
$$

In other words, they assumed that bidders' appraisals of costs, conditional on winning, are loglinear in their individual signals. Note that this makes the treatment of PCV here semiparametric, as it places parametric restrictions on the joint distribution of $(\boldsymbol{\eta}, V)$. Despite this additional assumption, $F_{\eta}(\eta)$ and $F_{V}(v)$ are still only partially identified up to the location and scale parameters $(C, D)$. However, this problem is less worrisome in light of the fact that any CIPI model is observationally equivalent to some CIPV model, meaning that any phenomena occurring within a PCV information structure may be modeled within a CIPV framework without loss of explanatory power; see Proposition 1 in Li et al. (2000).

The authors' estimation procedure combined methods developed by GPV with those of Li and Vuong (1998). Specifically, in the first stage of estimation they constructed a pseudo-sample of private signals using the inverse-bidding function and nonparametric estimates of the bid distributions. The second stage involves an application of nonparametric deconvolution methods to disentangle $F_{\eta}(\eta)$ and $F_{V}(v)$ from the sample of pseudo-signals. This same procedure applies equally to the recovery of the CIPV model or the partial recovery of the PCV model. Li et al. considered estimation of both models using a sample of OCS auctions conducted by the U.S. DOI.

Hong and Shum (2002) developed and applied a fully parametric approach to estimation in the CIPI model using a sample of auctions for road construction contracts conducted by the New Jersey Department of Transportation. An appealing characteristic of their model is that it has a built-in method to test between the polar extremes of PCV and IPV, but can also allow for elements of both to be present simultaneously. We follow up on this observation later in the special section on testing between common and private values.

Hong and Shum (2002) also adopted the log-additive specification of firm costs considered by Wilson (1998), although with some differences from Li et al. (2002). Here, firm costs have both idiosyncratic and common components, and are given by $C_{i}=A_{i} V$. Furthermore, firms do not directly observe their costs, but rather a noisy signal of costs $S_{i}=C_{i} e_{i}$, where $e_{i}$ is a positive error 
term. $A_{i}, V$ and $S_{i}$ are assumed to be log-normal, so that

$$
\begin{aligned}
\log S_{i} & =\log c_{i}+\varepsilon_{e_{i}} \sim N\left(\log c_{i}, \sigma_{e}^{2}\right), \\
\log V & =m+\varepsilon_{v} \sim N\left(m, \sigma_{v}^{2}\right), \\
\log A_{i} & =\bar{a}+\varepsilon_{a_{i}} \sim N\left(\bar{a}, \sigma_{a}^{2}\right) .
\end{aligned}
$$

Thus, the logarithm of private signals can be decomposed as

$$
\log S_{i}=(m+\bar{a})+\varepsilon_{v}+\varepsilon_{a_{i}}+\varepsilon_{e_{i}}
$$

and the parameters $\sigma_{v}^{2}, \sigma_{a}^{2}$ and $\sigma_{e}^{2}$ serve the double purpose of characterizing the joint distribution of signals (and hence driving bidder behavior), and parameterizing the relative importance of, respectively, the common-values component, the private-values component, and affiliation in the information structure. Hong and Shum showed informally using numerical examples that different combinations of values for these three parameters cause significant changes in both the shape and magnitude of the equilibrium bidding function. This, combined with the fact that variability in the number of bidders will also affect equilibrium bidding behavior, suggests that the model parameters are identified and estimable.

Hong and Shum (2002) employed a robust quantile estimator to recover the parameters of the latent signal distributions. The estimation procedure capitalizes on two important observations. The first is that the quantiles of a distribution $F_{S}(s)$ are invariant to monotonic transformations of $S$. The second is that equilibrium bids are merely monotonic transformations of $S$. Hence, the model parameters are chosen in such a way as to minimize a weighted average distance between observed bids and the equilibrium bid function evaluated at the quantiles of $F_{S}(s)$.

One of the authors' goals of their structural model was to uncover winner's curse effects in common-value auctions. Their estimates suggest a large winner's curse effect is present in the data. In auctions for highway contracts, as the number of bidders increases from two to ten, the median bid rises from $\$ 200,000$ to $\$ 350,000$. Moreover, the authors argued that this increase is entirely attributable to the winner's curse rather than the affiliation effect via a numerical exercise in which they shut down the common-values component while preserving other parameters at their estimated levels; see footnote 35 in their paper. This highlights one advantage of the above model specification. Namely, it achieves a high level of of explanatory power through explicitly separating the three components of the CIPI information structure (CV, IPV, and affiliation).

\section{Special Topics}

Our discussion thus far has partitioned the empirical auctions literature by informational paradigms nested within an AV structure. In this section we relax some of the assumptions that are maintained within canonical auction models and we try to deal with other econometric issues which, until this point, we have not discussed explicitly. As such, the structure of our presentation 
now shifts from one in which we focused on a given informational paradigm to one in which we focus on a specific topic. We try to discuss any work, regardless of the information structure, that relates to the topic of interest. Specifically, we address risk averse bidders, unobserved auctionspecific heterogeneity, accounting for an unobserved number of bidders, endogenous entry, dynamic aspects of auctions, testing informational paradigms, and auctions for multiple objects. Each of these topics have received particular attention in the literature.

Of course, there are a myriad of other special topics that could also have been included here such as bidder collusion, eBay, auctions for display advertising, scoring auctions, as well as numerous interesting structural econometric applications and case studies. The reader may think that our choice of which topics to cover was somewhat arbitrary: this point is well-taken and we offer as a defense the argument that the special topics we have chosen to include in this section are less specialized, which we feel allows for them to be considered in a number of different scenarios.

\subsection{Bidder Risk Aversion}

A maintained assumption in the papers discussed above is that a bidder's utility (if she wins) is simply her monetary surplus from the sale, or $u(s, p)=s-p$, where $p$ is the price she paid. However, if bidders are risk averse, then estimates based on this assumption will be biased and will lead to incorrect policy prescriptions. For example, it is well known from auction theory that riskaverse bidders bid more aggressively than risk-neutral ones. Therefore, a seller's mechanism design problem will be very different, depending upon which kind of bidders populate her auction. One well known example from auction theory is the fact that the first-price auction revenue dominates the Vickrey auction when bidders are strictly risk averse. This is because in the latter format truthful bidding is still a dominant-strategy equilibrium, while in the former risk-averse bidders will be willing to trade off some expected surplus to hedge against the risk of losing the auction. On the other hand, when bidders are risk-neutral, the revenue-maximizing mechanism is a Vickrey auction with an appropriately chosen reserve price. For these reasons, researchers have tried to adapt structural econometric methods to the case where $u$ is a concave von Neumann-Morgenstern utility function of unknown form. Donald and Paarsch (1996) were the first to consider identification of a model with risk averse bidders in a fully parametric setting. In our discussion here, we focus on recent work that has tried to identify models with risk averse bidders under minimal sets of assumptions.

Guerre, Perrigne, and Vuong (2009) studied nonparametric identification of first-price auction models within this setting, in which case equation (2) used by GPV to identify the risk-neutral model now becomes

$$
1=(\mathcal{N}-1) \frac{f_{B}(b)}{F_{B}(b)} \lambda(s-b),
$$

where $\lambda(\cdot)=u(\cdot) / u^{\prime}(\cdot)$. The authors show that equation (3) is insufficient to identify $\left(F_{S}, u\right)$ from observations of bids alone. In order to achieve identification in this more difficult setting, they adopt a strategy of including additional data on the number of bidders at auction. Specifically, they 
assume that the private value distribution is invariant to the number of bidders, so that participation in the auction must be exogenous. As it turns out, this assumption, which is standard in much of the literature, buys quite a bit of mileage: it implies that bidding strategies are strictly increasing in the number of bidders, so the quantiles of $F_{B}$ vary with $\mathcal{N}$ while those of $F_{S}$ do not. Intuitively then, for some $\alpha \in[0,1]$ one can compare the $\alpha$ quantile bid for auctions with $\mathcal{N}$ and $\mathcal{N}^{\prime}$ bidders to infer the level of risk aversion present. Remarkably, their results extend to much more general cases, such as binding reserve prices, APV, asymmetric bidders - where asymmetry may arise either from private signals in the usual way or from heterogeneous utility curvature - and even situations where $\mathcal{N}$ is endogenous, provided that suitable instruments are available.

While enlightening, one difficulty with the above method is that reliable measures of the number of bidders at auction are notoriously difficult to come by in practice (see our discussion below in the subsection on coping with unobserved numbers of bidders). Campo, Guerre, Perrigne, and Vuong (2011) developed an alternative approach for recovering bidders' private values and utility curvature in IPV, first-price auctions. Their analysis is based on an exploration of the minimal set of parametric restrictions necessary to identify the model from observations of bids. As it turns out, the nonidentification problem is somewhat deep: Campo et al. showed that specifying a parametric family for either the utility function or the private value distribution alone is insufficient to identify the model. However, they overcame this problem by adding another light assumption and including additional data that are more readily available in most contexts. Specifically, they assumed a functional form of the utility function while exploiting a parametric restriction of a single quantile of the private value distribution, in conjunction with data on auction-specific covariates to identify utility curvature. Moreover, they showed that dropping either of these two restrictions leads to loss of identification.

Now, estimation occurs in three stages. In the first, the conditional bid density is nonparametrically estimated at its upper boundary, being the quantile they chose to restrict in practice. In the second stage, the utility function parameters are recovered via NLS, using the nonparametric estimates from stage one. In stage three, the utility function estimate is plugged into equation (3) (where $F_{B}$ is now conditional on covariates) to recover a sample of private value estimates. Campo et al. established asymptotic theory for their estimator, showing that it converges at the fastest possible rate for any semiparametric estimator of the model, which is the usual $\sqrt{T}$ rate if $u$ is analytic, and slightly slower otherwise. They also demonstrated how their method can be adapted to handle bidder asymmetry, APV, or binding reserve prices. They applied their method to a sample of US Forest Service timber auctions, and found evidence for significant levels of risk aversion among participants.

Lu and Perrigne (2008) sought to avoid the necessity of imposing additional identifying assumptions for recovery of bidder preferences by exploiting the availability of data from both English and first-price auctions for similar objects. Given the availability of such rich data, the econometrician may employ a fully nonparametric approach to estimating bidder risk aversion. The intuition here is that truthful bidding is an equilibrium in dominant strategies for the English auction, regardless 
of risk aversion, whereas in the first-price auction, risk-averse bidders will bid more aggressively than will risk-neutral ones, so as to hedge against the risk of losing the auction. Lu and Perrigne proposed using data on winning bids from English auctions to nonparametrically recover private value estimates, which then can be plugged into equation (3) to nonparametrically identify $u$. Note, however, this procedure requires that the same set of bidders participate in both types of auctions, otherwise estimates from the first and second stages are incompatible. Lu and Perrigne estimated the model using a sample of US Forest Service timber auctions for which both pricing mechanisms were used. They also used a semiparametric adaptation of the estimator to asses how well some popular utility specifications fit the data, finding that CRRA provided a reasonable fit, while the CARA specification did not.

\subsection{Unobserved Heterogeneity}

Often auction researchers assume that they have access to the same information about the items at auction as the participants at the auctions. As such, variation in observed bids across objects that are considered to be the same (after conditioning on observables) is attributed to variation in bidder valuations. In reality, bidders may have access to some information about the object (or project in a procurement setting) being auctioned which may not show up in the data and, hence, is unobserved to the econometrician. Failing to account for this unobserved heterogeneity may bias estimates of the structural parameters or the unconditional empirical distribution of bids, which can propagate through the model and lead to incorrect inference concerning bidder valuations and, hence, poor policy recommendations. While some authors have attempted to deal with unobserved heterogeneity (for example, the issue was recognized by Laffont et al. (1995)), we describe in this subsection researchers that have put unobserved heterogeneity at the center of their work and have proposed ways of dealing with it explicitly. Note, too, that the issue is inherently an econometric one-it's not a modeling issue or of importance to theorists.

Some previous researchers considered unobserved heterogeneity but it was tightly linked with bidder participation; see, for example, Hong and Shum (2002). In such settings, the researcher may control for unobserved characteristics by conditioning on the bidder makeup of an auction (i.e., the number of total bidders, the number of bidders of different types, etc.). Campo et al. (2003) suggested a means for testing for unobserved heterogeneity along these lines. Remember that the authors considered OCS wildcat auctions with two types of bidders: solo and joint bidders. The behavioral nature of cartel formation by joint bidders provides a basis for a test of unobserved heterogeneity. Here, the underlying idea is that there are a set of characteristics observable to bidders which motivate their decisions to form cartels or to act on their own. Campo et al. assumed essentially that bidder makeup in an auction forms a sufficient statistic for these characteristics, which are unobservable to the econometrician. It follows then that a test for the presence of unobserved heterogeneity may be afforded by a Kolmogorov-Smirnov test applied to the marginal densities of joint bidders estimated from auctions in which they face solo bidders and also when they face other joint bidders. If these densities differ significantly, then the researcher may reject 
the null hypothesis of no unobserved heterogeneity. Finally, the authors argued that additional evidence may be found by examining and comparing estimates of information rents across auctions with different bidder makeups.

Krasnokutskaya (2011) provided the first characterization of a model with auction-specific unobserved heterogeneity. She focused on identification and estimation, and even allowed for unobserved heterogeneity to be present across auctions with the same profile of bidders. Krasnokutskaya modeled bidders' values as the product of a private component and a common component which is observable to bidders but not to the econometrician. If these two components are independent of each other and, when multiplied, comprise the bidders' valuations, identification obtains as long as two bids are observed at each auction. By assuming multiplicatively-separable components, she was able to relate her work to and leverage results from Li and Vuong (1998) as well as Li et al. (2002). Krasnokutskaya cleverly reduced the econometric problem to a measurement error problem with multiple indicators, as first studied by Li and Vuong; she also invokes the asymptotic properties of the estimators they established.

A difficulty obtains in the presence of unobserved heterogeneity: the first-order conditions express valuations as a function of both bids and the realized value of the unobserved heterogeneity. A consequence of this is that the standard GPV identification results based off the first-order conditions cannot be applied. Instead, Krasnokutskaya focused on the joint distributions of bids submitted in the same auction. The marginal density of the unobserved heterogeneity can be identified from the joint distribution of two bids sharing the same common valuation (cost) component. Intuitively, the component corresponding to unobserved auction heterogeneity induces dependence among bids at a given auction and this dependence can be used to recover the marginal distributions under some independence assumptions (the bidders' individual valuation/cost components are independent of each other and of the common component). In estimation, Krasnokutskaya followed her identification strategy by estimating the joint characteristic functions of bids and then used a deconvolution technique to separate out the densities of the common and private bid components; that is, the marginal distribution of unobserved heterogeneity and of bids, respectively. She then used the bid densities to estimate nonparametrically the distribution of the individual valuation (cost) component much in the same way as GPV. After providing Monte Carlo evidence that her estimation procedure performs well, she applied these techniques to a sample of Michigan Department of Transportation highway procurement auctions. She showed that unobserved heterogeneity may be of significant concern in practice and is an important one for optimal auction design.

The approach taken by Krasnokutskaya employed results from the classical measurement error literature: with multiplicatively-separable components, equilibrium bids scale multiplicatively in the unobserved heterogeneity (or, taking logarithms, the log of unobserved heterogeneity acts as an additive mean shifter of the distribution of the $\log$ of bids for a given item). In contrast, $\mathrm{Hu}$, McAdams, and Shum (2010) proposed an approach that is rooted in the more recent literature on nonclassical measurement error. The authors provided an approach for identifying bidder valuations in an asymmetric IPV framework in the presence of unobserved heterogeneity. Their approach 
is more flexible than Krasnokutskaya's in that the unobserved heterogeneity can affect bidders' valuations in a nonlinear way, but requires observation of a third instrument (in addition to the two bids) at each auction. In the case where this instrument is a bid, three bids must be observed at each auction - the third bid plays the role of an instrument for the unobserved heterogeneity. To identify their model the authors assumed there is a monotone relationship between bids and the unobserved heterogeneity. Whereas Krasnokutskaya (2011) required this relationship be multiplicative, here the effect can be non-separable as well as asymmetric on the different bidders. The assumption allowed the authors to identify the distribution of the unobserved heterogeneity as well as the conditional distribution of bids, given the unobserved heterogeneity, from the observed joint distribution bids alone. The intuition is that this joint distribution is decomposed into a mixture of conditionally independent bid distributions (hence, it would be difficult to allow for affiliation using this strategy). Of course, the distributions of valuations, conditional on the unobserved heterogeneity, is identified from the conditional distributions of bids. The authors discussed settings in which monotonicity concerning unobserved heterogeneity obtains naturally (such as product quality, reservation price, bidding cost, or the number of bidders) and suggested a nonparametric sieve ML estimation procedure.

While many researchers leverage changes in the number of bidders at auction to help identify any unobserved heterogeneity, Roberts (2011) offered a novel approach by exploiting choices made by the seller - who is assumed to observe all auction characteristics - to identify a symmetric, IPV, English auction model in the presence of unobserved heterogeneity. To do this, he modeled sellers who set reserve prices that are not necessarily optimal by assuming a relationship between the reserve price and unobserved heterogeneity: higher realizations of unobserved heterogeneity induce the seller to set a higher reserve price. Doing so allowed him to identify the distribution of valuations conditional upon observables and the realized unobserved heterogeneity from observing data on winning bids and reservation prices alone. In estimation, an econometrician would first use the reserve prices and auction covariates to estimate the distribution of unobserved heterogeneity and a reserve price function (used by sellers); in a second step, the distribution of valuations can be estimated given the observable covariates and the implied realization of the unobserved heterogeneity from the first-step estimation. He studied an application in which unobserved heterogeneity is likely important and his approach attractive: used car auctions from South Korea for which the number of bidders at each auction is unknown. After estimating his model semiparametrically (he imposed structure on the reserve price function but estimated valuations in the second step nonparametrically), Roberts argued that too much emphasis is put on identifying the optimal reserve price and too little put on the value of adding another bidder.

Aradillas-López, Gandhi, and Quint (2011) noted that real-world auction data often contain heterogenous objects and researchers who assume independent private values must condition estimates of the latent private value distribution(s) on observable covariates. However, such a strategy does not adequately address any unobserved heterogeneity which might affect demand for the objects at auction. Specifically, these unobservables might cause bidders' valuations to be correlated 
and this has important implications for optimal auction design. The authors obtain identification of a model with correlated private values in which private values are independently and identically distributed draws from a distribution that depends on observable as well as unobservable covariates. The authors showed that the unobservables introduce correlation into private values, and moreover, when the correlation is properly accounted for the implied optimal reserve price suggested by an IPV model would be too high.

Aradillas-López et al. considered U.S. Forest Service data from ascending timber auction data. Krasnokutskaya's (2011) approach for a first-price setting allowed her to observe multiple bids for a given auction which she could exploit given her structural model. A difficulty with English auctions is that equilibrium bidding places structure on only one bid (and often only one bid is observed). Aradillas-López et al. leverage variation in winning bids across auctions with different numbers of bidders (different size) to partially identify their model. Specifically, assuming valuations are stochastically higher in auctions with more bidders allowed them to construct an upper bound on the seller's expected profit, while assuming valuations are independent of the number of bidders provides a lower bound. The authors then introduced a nonparametric test with known asymptotic distribution to decipher which assumption is reasonable. Their method allows researchers to conduct estimation and inference even if the covariates a researcher includes in her model do not account for all heterogeneity across auctions.

\subsection{Unobserved Number of Bidders}

Applied econometricians often assume that the number of potential bidders at auction equals the number of observed bidders; however, this can be difficult to justify when data come from auctions in which reserve prices were used or settings in which bidders faced some type of entry cost. Unfortunately, reliable measures of the total number of potential bidders in an auction are rare in practice, yet these are critical for empirical work as they are needed to characterize the data generating process for bids.

Paarsch (1997) achieved a breakthrough in this area while studying English auctions for BritishColumbian timber. Given a set of $\mathcal{N}$ potential bidders and reserve price $r$, we know that bidders only participate in bidding if their value, $s$, exceeds the reserve price $r$. Moreover, in an English auction, bids are equal to values. Thus, the joint density of the number of actual participants, denoted $N$, the ordered set of $N$ observed values and the $(\mathcal{N}-N)$ unobserved values is

$$
\begin{aligned}
f_{\mathbf{S}(N), N}\left[s_{(1: \mathcal{N})}, \ldots, s_{(n-1: \mathcal{N})},\right. & \left.s_{(n: \mathcal{N})}, \ldots, s_{(\mathcal{N}: \mathcal{N})}, n\right]= \\
& \left(\begin{array}{c}
\mathcal{N} \\
n
\end{array}\right) F_{S}(r)^{(\mathcal{N}-n)} n ! \prod_{i=1}^{n} f_{S}\left(s_{(i: \mathcal{N})}\right),
\end{aligned}
$$

where the $n$ ! counts all the ways in which $n$ values could be ordered, and the $\left(\begin{array}{l}\mathcal{N} \\ n\end{array}\right)$ counts all the ways in which $n$ of $\mathcal{N}$ values could be chosen to have a value greater than $r$. Paarsch s innovation was in noticing that part of this expression bares close resemblance to the binomial density of the 
number of participants, or

$$
f_{N}(n)=\left(\begin{array}{c}
\mathcal{N} \\
n
\end{array}\right) F_{S}(r)^{(\mathcal{N}-n)}\left(1-F_{S}(r)\right)^{n}
$$

Thus, by multiplying the right-hand side of 40 by $\frac{\left(1-F_{S}(r)\right)^{n}}{\left(1-F_{S}(r)\right)^{n}}$, one can separate out the probability of observing $n$ participants, from the joint density of their ordered values, conditional on $n$, which is

$$
n ! \prod_{i=1}^{n} \frac{f_{S}\left(s_{(i: \mathcal{N})}\right)}{\left(1-F_{S}(r)\right)} .
$$

Note that an expression like (5) can easily be incorporated into a conditional ML estimation scheme with no precise knowledge of $\mathcal{N}$. This proves a useful contribution from a policy perspective, as it allowed Paarsch to construct an estimate of the optimal reserve price in timber auctions conducted by the Canadian government. He concluded that historical reserve prices have been lower than optimal and, interestingly, the Canadian government has since nearly doubled its reserve prices for timber 8

Like Paarsch's work, much of the research addressing the number of potential bidders is concerned with second-price auctions or, perhaps, more appropriately, electronic auctions 9 Specifically, electronic auctions like eBay and Yahoo! involve bidders vying for an object from their own computer. It's difficult to gauge how many potential bidders there might be, but this is important in characterizing the underlying valuation distribution despite the weakly dominant bidding strategy of bidding one's valuation. The issue arises because econometricians often only observe (or can only trust) two bids in such settings. Moreover, these bids have important meanings: they are (often) the second- and third-highest bids. Thus, pooling these bids across auctions and estimating the latent valuation distribution from them is not possible because they constitute order statistics from a distribution in which the number of draws might be changing across auctions.

Song (2004a) was the first to recognize formally and address these concerns. Specifically, she modeled an electronic auction as a second-price auction in a symmetric IPV framework. She showed that the bidders' valuation distribution is identified nonparametrically if the researcher observes any two valuations and knows their rank ordering, relative to the highest bid (for example, the researcher sees two bids and knows they represent the second- and third-highest valuations). The key to her identification argument is a lemma in which she showed the distribution of two order statistics, from an iid sample, uniquely determines the parent distribution - even if the size of each sample is unknown and varies across observations. Intuitively, since Athey and Haile (2002) showed the distribution of one order statistic from a sample of known size identifies the parent distribution, observing a second draw allows one to characterize a truncated distribution by conditioning on the

\footnotetext{
${ }^{8}$ See page 77 of Paarsch and Hong $(2006)$.

9 Hickman, Hubbard, and Paarsch (2011) provide an econometric analysis of the commonly-adopted modeling assumption that electronic auctions are second-price auctions. Specifically, they found that the assumption can lead to significantly different parameter estimates but that the policy prescriptions stemming from the assumptions lead to negligible economic differences. Moreover, they showed electronic auctions are nonparametrically identified and suggested a simple modification involving a correction term to account for the presence of minimum bid increments.
} 
lower of the draws from a sample of known size (two).

Specifically, Song (2004a) studied an equilibrium in which the two bidders with the highest valuations always bid their valuation. She proposed a formal test and an estimation strategy of when the bidder with the third-highest valuation actually bids her valuation based on how late in the auction the first- or second-highest valuation bidders outbid her. In estimation, Song proposed using a seminonparametric approach in which the valuation density is assumed to be a series of Hermite polynomials, the coefficients of which are estimated via ML estimation. She illustrated her method using Monte Carlo experiments as well as a sample of auctions for university yearbooks on eBay, for which she estimated the consumer surplus bidders obtained at the auctions. Sailer (2006) extended the approach of Song to an asymmetric second-price setting but required that the researcher observe bidder identities as well. Her key observation was that focusing on the distribution of the second-order statistic conditional on the third-order statistic not only makes the object of interest independent of the number of potential bidders, but also the distribution functions from which these rival bidders draw valuations.

In first-price models, Song (2004b) extended her ascending-auction work to a symmetric firstprice setting in which the econometrician observes the first- and second-highest bids which can be leveraged to identify the valuation distribution as well as the distribution of the number of potential bidders. Her estimation methodology follows the two-step approach of GPV in which she modifies the first step to account for the fact that she observes just the maximum of each the rival bids since she observes the two highest bids at auction. While Song (2004b) assumed that bidders do not observe the number of active bidders, more recently, An, Hu, and Shum (2010) considered a first-price model in which bidders observe the number of potential bidders $\mathcal{N}$, but the econometrician does not. The authors considered a misclassification approach to identify and estimate nonparametrically the model under this assumption. Allowing the number of potential bidders to vary across auctions implies that observed bids are drawn from a mixture distribution with unknown weights. As such, the authors leveraged deconvolution methods (like Krasnokutskaya (2011) did in a different context) developed in the econometric literature on measurement error. Their contribution can also be thought of as modifying the first step of the two-step nonparametric estimation procedures. Specifically, the first step requires estimating a conditional density as observed bids are essentially truncated because of the presence of a reservation price. To do so, An et al. required two auxiliary variables: a mismeasured variable for the number of potential bidders for which the authors used the number of actual bidders and an instrument which they took to be a discretized second bid from each auction. These provided the authors with a matrix equation which is an eigenvalue-eigenvector decomposition of a matrix representing something observed in the data. The authors presented Monte Carlo evidence that accounting for the unknown number of potential bidders is important and that their method works well. They then applied their estimator to procurement data from New Jersey and showed that accounting for the unobserved number of potential bidders leads to differences in bidders' informational rents.

A direct way to obtain differences in the number of observed bidders and the the number of 
potential bidders is to model explicitly the bidders' decision to participate in an auction. In the next subsection, we consider research that deals with endogenous entry.

\subsection{Endogenous Entry}

Most researchers consider models in which the number of bidders is given or fixed. However, if entry is endogenous then the number of active bidders is stochastic. Furthermore, in the presence of reservation prices a truncation problem arises: bids are only observed from players whose valuations are at least as high as the reserve price at auction. As such, the number of actual bidders is endogenous and the number of potential bidders is unobservable. This poses as problem as consistency may no longer hold for standard models as the equilibrium bid functions depend on this unobserved quantity. Moreover, the entry problem is likely to be quite common: Li and Zheng (2009) reported that only $28.05 \%$ of the bidders requesting official project proposals (which can be thought of as the set of potential bidders) actually submitted bids.

Li (2005) studied estimation and identification in a IPV model in which bidders must incur an entry cost in order to observe a private valuation (thus becoming a potential bidder), and then, in the second stage pf the game, they bid optimally at a first-price, sealed-bid auction by following a symmetric Bayes-Nash equilibrium strategy. The second stage involves auctions with known reservation prices and so, even after incurring and initial cost, some bidders may not submit bids. Li developed moment conditions concerning the observed bids and the number of actual bidders which take into account the effects of entry and the binding reservation prices. He proposed a method-ofsimulated-moments approach in the spirit of the simulated NLS approach of Laffont et al. (1995), as opposed to a generalized MOM estimator which would require computing the equilibrium bid function for each trial parameter vector. However, Li s method-of-simulated-moments approach has advantages over that of Laffont et al. (1995), as the number of bidders can vary across auctions and it does not require a bias correction to be consistent for a fixed number of simulations. In addition to providing a way to estimate simultaneously the structural parameters in both the bidding and entry processes, Li provided formal tests of binding reservation prices, of validity of the structural approach (the theoretical model), and of mixed-strategies of entry (as he assumed that after incurring a cost each bidder entered the auction with some probability). Li did not consider an empirical application but used Monte Carlo experiments to show the importance of taking into account the entry process in estimation.

Li and Zheng (2009) used data on highway procurement contracts (mowing services) from the Texas Department of Transportation to estimate three models of endogenous entry. The authors, like $\mathrm{Li}$ (2005), considered a symmetric IPV framework but expanded the set of entry models to include: (i) a mixed-strategy entry model in which bidders first decide whether to incur a common cost in order to acquire a signal and submit a bid; (ii) a pure-strategy entry model in which bidders first decide whether to incur a bidder-specific entry cost, drawn from a common distribution of entry costs, in order to acquire a signal and submit a bid; (iii) a pure-strategy entry model in which bidders learn their signal before deciding whether to enter the auction. While the models differ in 
their assumptions concerning the timing and informational structure of the entry game, all of them yield some simple comparative static predictions. For example, in all models, as the number of potential bidders increases, all else equal, equilibrium bids are more aggressive (the "competition effect"), but the probability of entering the auction decreases and, since for a fixed number of potential bidders this implies less actual bidders in expectation, an "entry effect" predicts the opposite sign. As a result, the relationship between the equilibrium bids (or the expected winning bid) and the number of potential bidders may be positive or negative (in all models) depending on the size of these effects. To estimate the models structurally the authors proposed using recent semiparametric Bayesian methods to recover the entry cost distribution as well as the bidders' cost distribution (parametrically) and the distribution of unobserved heterogeneity (nonparametrically). Specifically, the authors leveraged data augmentation and Markov chain Monte Carlo methods to develop an estimation algorithm and then used mean-squared errors of the predictions to select the model that best fits the data. The authors found the first model, accounting for unobserved heterogeneity, fit the data best and used the structural estimates to conduct counterfactual analysis by quantifying the effects of changing the number of potential bidders on the individual bid as well as on the procurement cost. The authors found the positive entry effect dominated the negative competition effect and, as such, policies which encourage more potential bidders might actually increase costs for the government! An implication of their findings is that policies that reduce entry costs will save the government money whereas policies that increase the number of potential bidders might actually cause procurement expenses to increase.

Given the opposing competition and entry effects, it suggests there may be an optimal number of potential bidders. Zheng (2009) investigated the empirical importance of missing this optimal target using a sample of Michigan Department of Natural Resources timber auctions. Specifically, he estimated the structural parameters of the entry model selected by Li and Zheng (2009), although he favored the model without unobserved heterogeneity in his application, using an iterative multistep ML estimation procedure. Specifically, the first step involves a likelihood function based only on the number of actual bidders, the second step recovers private values, and the third step considers the likelihood of a truncated density. Zheng then used his parameter estimates to calculate the optimal number of potential bidders at each auction he observed in his timber auction data. He simulated auction outcomes under the optimal policies to compute the increase in the winning bid as well as social welfare - defined as the sum of the seller's expected revenue and the bidders' expected profits - finding the loss due to market thickness (too many potential bidders) to be on the order of $3 \%$ of the median winning bid he observed.

Li and Zheng (forthcoming) considered a unified Bayesian approach in which the two canonical entry models could be estimated and distinguished. Specifically, a key distinction between models of endogenous entry is whether signals are learned before (in which case entry costs might be interpreted as bid preparation costs) or after (in which case the entry costs would constitute both signal acquisition and bid preparation costs) the decision to enter the auction is made. Like Li and Zheng (2009), the authors used Bayesian methods in estimation. One contribution of this work 
is that they used (log) Bayes factor to compare different models' fits using the same data. The Bayes factor can be thought of as the posterior odds in favor of one model over another, given both are equally probable a priori; or, alternatively, it can be viewed as the relative success of two models at predicting the observed data. They applied their approach to Michigan Department of Natural Resources timber data and selected the model in which signals are learned before the decision to enter the auction is made (they found "very strong" evidence against the model in which signals are learned after the entry decision). They used this model to quantify the entry and competition effects, to estimate the optimal reserve price, and to measure gains if an optimal policy were used. Interestingly, here they found the competition effect dominates the entry effect suggesting the auctioneer should encourage potential bidders.

Krasnokutskaya and Seim (forthcoming) considered an empirical study of bid preference policies used under the California Small Business Bidding Preference Policy to estimate a model of bidding and endogenous entry as well as to consider alternative policy designs. The authors argued, and found, that programs which treat bids asymmetrically for evaluation purposes can induce effects on firms' incentives to participate in an auction. As such, any evaluation of bid preference programs that treat participation as fixed can yield misleading results. The authors considered an entry model in which potential bidders do not observe cost (or valuation) realizations at the time in which they decide whether or not to enter the auction. In estimation, the authors accounted for unobserved heterogeneity by using the approach of Krasnokutskaya (2011) and parameterized the distributions of the bidder-specific bid component and the distribution of the unobserved heterogeneity (both of which were assumed to be log-normal) as well as the distribution of entry costs (which was assumed follow a left-truncated normal distribution). The authors used a first-step simulated generalized MOM estimation approach and adopted the nonparametric second-step of GPV in considering highway and street maintenance projects let by the California Department of Transportation. Krasnokutskaya and Seim found that the current bid preference program generates only small increases in procurement costs but does not achieve the state's allocation goal concerning the dollar-share of contracts awarded to small businesses (a higher preference rate is required and raises costs of procurement by 1.4 percent relative to no intervention).

Li and Zhang (2010a) considered estimation of an asymmetric first-price model with endogenous entry in the APV paradigm. The authors extend the approach of Hubbard et al. (forthcoming) by employing a copula-based approach but estimation is complicated by the presence of asymmetric bidders as well as endogenous entry. To accommodate these features the authors resorted to a fully parametric approach by assuming the marginal distributions of private values to be truncated exponential distributions and the distributions of entry costs to be (untruncated) exponential distributions, each of which are linked by a Clayton copula to construct the joint valuation and entry cost distributions which have different dependence parameters. The authors estimated their model via indirect inference. Specifically, the optimal parameters were found by generating data from their model for a given parameter choice and minimizing the distance between parameters of an auxiliary model using the original and simulated data. Of course, simulating data from an asymmetric 
model is complicated by the fact that the inverse-bid functions cannot be computed analytically and require numerical methods. Li and Zhang's auxiliary model included two regressions: a linear model concerning the average bids and a Poisson model concerning the number of actual bidders, both of which used the same set of auction-specific covariates. The authors estimated their model using timber sale auctions conducted by the Oregon Department of Forestry and found small, but significant, dependence in the private signals but much higher dependence in the distribution of entry costs. They conducted counterfactual analyses to consider the effects of different reservation prices and dependence levels as well as the effects of merger activity among the bidders.

Athey, Levin, and Seira (2011) recently provided a nice case study which links many of the issues and methods we've discussed in our survey, which are often studied in isolation. The authors observed differences in expected revenues and participation across English auctions and first-price, sealed-bid auctions at U.S. Forest Service auctions for timber near the Idaho-Montana border, and in California. They constructed a private values model in which bidders incur information acquisition costs (bidders must pay a random entry fee in order to observe their private value) and draw valuations from asymmetric distributions to reconcile the differences as, in a symmetric IPV model, theory predicts that auction format has no effect on entry or revenue. However, when bidders are asymmetric the open, ascending auction format discourages entry by "weak" bidders, as competitors with the highest value always win 10 In contrast, first-price auctions provide "strong" bidders with incentive to shade bids when competing against weak bidders, and the possibility of inefficient outcomes encourage entry by weak bidders, as it shifts allocations in their favor; see Maskin and Riley (2000a). The asymmetry assumption is attractive as there are likely two types of bidders at these auctions: loggers (small firms with low capacity) and mills (larger firms with high capacity). Lastly, the authors also allowed bidders to cooperate in the English auctions - a setting where collusion is of concern given participants can witness and react to their partners' actions.

Athey et al. constructed a structural econometric model of first-price auctions within the IPV paradigm with asymmetric, risk-neutral firms to achieve three goals: (i) to see whether the estimated model predicts expected revenues that are different across auction formats; (ii) to measure how competitively bidders behave in the open auctions; (iii) to investigate welfare implications under the different auction formats. The authors used a parametric version of GPV to recover the distributions of bidder valuations and followed Krasnokutskaya (2011) to account for unobserved heterogeneity in the sale characteristics. Specifically, the authors abandon the nonparametric approach of the first stage in favor of a Gamma-Weibull specification of bids and unobserved heterogeneity. Note that, to apply the GPV estimator, one can specify either the bid distribution and estimate the valuation distribution nonparametrically or specify the valuation distribution and estimate the bid distribution nonparametrically. However, a researcher cannot make parametric assumptions at both stages or an internal inconsistency will obtain in the bidding strategies. The second stage was also complicated by the fact that the distributions of valuations depend on un-

\footnotetext{
${ }^{10}$ The authors assumed that there are two types of bidders, "weak" and "strong." The former are so named because the distribution of their private values is assumed to be stochastically dominated by that of the latter. This is standard language used by auction theorists.
} 
observed heterogeneity. By averaging over realizations of unobserved characteristics, the authors estimated the typical markup for weak and strong bidders. In their model, the distribution of logger entry is binomial and the authors estimated the probability of entry by ML using observed logger entry patterns and a multinomial logit model. An attractive feature of their data is the variation in auction format, which is what allows the authors to asses the competitiveness of the English auctions by using out-of-sample predictions based off the first-price estimates which are compared with the actual outcomes. They found that asymmetries alone led to little differences in the properties of the different auction formats, but that when endogenous entry was accounted for, the first-price format favored the loggers (small bidders).

\subsection{Auction Dynamics}

In this section we briefly discuss models of repeated bidding where time dynamics play a prominent role. These models are best thought of as special cases of the recent literature on estimation of dynamic games, but where estimation of an auction model is of primary interest. The papers we discuss in this section are distinct from auctions for multiple objects in that there is typically a state variable - e.g., production capacity in procurement settings - that imposes constraints on bidding in later rounds.

Jofre-Bonet and Pesendorfer (2003) estimated a model of infinitely repeated (i.e., sequential), first-price procurement auctions with bidder capacity constraints to capture the essential features of highway construction procurement. They constructed a dynamic model to account for the effects of previously won but not completed projects. These have a two-fold effect. On the one hand, they may occupy some capital resources (e.g., machinery) so costs increase. On the other hand, these contracts increase firms' experience in highway construction, thus leading to a reduction in costs. As a result, they may lead to some inefficiencies. To investigate which effect would dominate, they set up a first-price procurement auction model, where bidders are asymmetric during periods in which they stay in the game. Regular bidders stay forever, while fringe bidders enter the game for one period and then exit. They adopted numerical methods to approximate the value function of a firm, and for estimation they proposed a two-stage estimator that builds off of ideas developed in Elyakime, Laffont, Loisel, and Vuong (1994) and GPV. In the first stage they computed the bidding function conditional on the state variables, and in the second stage they computed both the expected sum of future profits conditional on the distribution of bids as well as the costs using the first-order condition from the dynamic model. They concluded that the first effect explained above dominates the second effect, thus leading to an increase in firms' costs.

Ji and Li (2008a) exploited a multi-round feature of procurement auctions conducted by the Indiana Department of Transportation. Specifically, if a contract is not awarded (perhaps because all bids exceed a price ceiling in a low-price setting) the government may re-auction the contract in additional rounds. The authors constructed a dynamic multi-round model in which bidders account for the possibility of the contract being reauctioned when setting their bids in a given round. The auctioneer sets a secret reserve price which bidders learn about through bidding rounds - they know, 
for example, that if the contract goes unawarded the reserve price is below the lowest submitted bid. As such, bidding gets more aggressive over time. They estimated their model under the assumption that bidders act myopically which allows the dynamic model to be treated like a static model. Their structural model includes three primitives to be estimated: a distribution characterizing the government's reserve price as well as two private cost distributions, one for each of the two rounds they observe in their data. While these distributions are identified nonparametrically under their assumption that bidders are not forward looking, the authors opted for a parametric approach since they did not observe many second-round auctions.

Ji and Li considered estimation in two stages. First, they used a simulated ML approach to recover the parameter characterizing the government's reserve price distribution and a parameter corresponding to unobserved heterogeneity which they accounted for in their application. The unobserved heterogeneity prevents a closed-form likelihood function which the authors dealt with by using importance sampling in numerical integration. In the second step, they estimated the firstand second-round cost distributions using moment conditions, here using a method of simulated moments to deal with unobserved heterogeneity. The authors fail to reject the hypothesis that cost draws come from different distributions. They also investigated whether it was best to hide or announce the reserve price, finding that it depends on the specification of the latent cost distribution. In their data they found that keeping the reserve price secret could allow cost savings relative to the case where it is disclosed.

\subsection{Testing Informational Paradigms}

A critical modeling choice that the structural researcher must make concerns the information paradigm. A key distinction on which the literature has concentrated is that between common versus private values. Does a bidder's private information correspond to her idiosyncratic preference for the good, or is it a signal of an unknown value, which could be refined if she had access to her rivals' private information? These distinctions have a profound impact on counterfactual results and policy prescriptions. Even more generally, as evident from our discussion in this survey, nearly all structural econometric work has taken place within the general AV model. As such, researchers may wish to provide evidence that implications of an AV model are consistent with their data. In this subsection, we first discuss testing between common and private values and then we discuss recent work that has considered testing for affiliation.

\subsubsection{Testing Common versus Private Values}

Three noteworthy papers employ parametric methods to distinguish between common- and private-values paradigms. To our knowledge, Paarsch (1992) was the earliest attempt. Recall from the above discussion that Paarsch derived the data generating process under several different specifications of the latent variables for both the IPV and PCV models. This derivation included the distribution over winning bids as well as expressions for the raw population moments of winning bids. Two alternative estimation methods are implemented: a ML estimator, using the density of 
winning bids to construct the likelihood function; and a NLS estimator, using an expression for the $r^{\text {th }}$ raw moments given by $W^{r}=R_{r}(p)+e$. The testing procedure which Paarsch (1992) proposed first estimates the IPV and PCV models using both methods and then seeks to rule out cases according to different criteria. Within a particular paradigm, a Hausman (1978) type test may be used to evaluate whether the ML and NLS estimates are close to one another. Alternatively, a White (1982) test may be applied to the ML parameter estimate(s). If neither CV nor PV are rejected by these tests, then the non-nested hypothesis testing methods of Cox $(1961,1962)$ can be applied to decide between IPV and PCV. Alternatively, the methods of MacKinnon, White, and Davidson (1983) may be used to overcome the heteroskedasticity and non-normality of the NLS errors.

As discussed in the Section 6 on the CIPI paradigm, Hong and Shum (2002) constructed a parametric empirical specification with a built-in test of PCV versus IPV. Recall that Hong and Shum (see section 6 of this survey) assumed that firm costs have both idiosyncratic and private components and that they adopted Wilson (1998) log-additive specification of firm costs for tractability. Thus, costs are given by $C_{i}=A_{i} V$. Furthermore, recall that firms do not directly observe their costs, but a noisy signal of costs $S_{i}=C_{i} e_{i}$, where $e_{i}$ is a positive error term. $A_{i}, V$ and $S_{i}$ are assumed to be log-normal, so that

$$
\begin{aligned}
\log S_{i} & =\log c_{i}+\varepsilon_{e_{i}} \sim N\left(\log c_{i}, \sigma_{e}^{2}\right), \\
\log V & =m+\varepsilon_{v} \sim N\left(m, \sigma_{v}^{2}\right), \\
\log A_{i} & =\bar{a}+\varepsilon_{a_{i}} \sim N\left(\bar{a}, \sigma_{a}^{2}\right) .
\end{aligned}
$$

Hence, log private signals can be decomposed as

$$
\log S_{i}=(m+\bar{a})+\varepsilon_{v}+\varepsilon_{a_{i}}+\varepsilon_{e_{i}}
$$

and the parameters $\sigma_{v}^{2}, \sigma_{a}^{2}$ and $\sigma_{e}^{2}$ serve the double role of characterizing the joint distribution of signals (and hence driving bidder behavior), and parameterizing the relative importances of, respectively, common-values components, private-values components, and affiliation in the information structure. It is this latter role that is relevant to model testing. Once the variances of the three types of errors are recovered from their proposed quantile estimation method (see Section 6 above), their standard errors may also be calculated, after which standard t-tests then reveal the significance of common-value components, private-value components and affiliation in the information structure. Thus, an appealing characteristic of their model is that it has a built-in method of testing between the polar extremes of PCV and IPV, while allowing for elements of both to be present simultaneously.

Bajari and Hortaçsu (2003) studied eBay auctions, which they argued correspond to the English auction format. They were motivated by features of the sale objects in their data - rare collectible coins with unknown future resale value - to assume a CV information structure. However, to be sure they proposed a simple reduced-form test based on results proven by Milgrom and Weber (1982), 
who pointed out that in English auctions, bids based on private values are unaffected by changes in competition, whereas CV bids are decreasing in the number of bidders. This distinction can be easily verified by regressing bids on the number of bidders $\mathcal{N}$ and performing simple hypothesis tests on the coefficient of $\mathcal{N}$.

Several other papers have attempted to test between common and private values without imposing parametric assumptions. Athey and Haile (2002) proved sets of sufficient observables that will allow for nonparametric testability of private values models against the common values alternative (see Theorems 9, 10, 11 and 12). Another important theoretical econometric paper by Laffont and Vuong (1996) informs the model testing endeavor by providing insights into how the AV subcases relate to one another. As shown in their Proposition 1, if the number of potential bidders is held constant, then any model within the symmetric AV framework is observationally equivalent to some symmetric APV model. This means that any symmetric common values model is empirically indistinguishable from some symmetric private values model on the basis of bids alone. Moreover, (see Laffont and Vuong (1996, Proposition 9)), when the number of bidders is held constant, any asymmetric CV model is also observationally equivalent to some asymmetric IPV model. These two surprising results imply that unless the number of observed bidders varies, it is impossible to distinguish between private values and common values models, without imposing parametric restrictions on the model. For this reason, tests of common versus private values that were developed after Laffont and Vuong (1996) were mostly based on observations of varying numbers of bidders across different auctions.

Campo et al. (2003) discovered a method for testing the appropriateness of the PCV information structure when bidders are asymmetric. Since in a PCV model it is only bidders' signals of value which differ, it turns out that bidding strategies do not actually differ across bidder types. This leads to their Proposition 2, which states that a sample of observed bids can be rationalized by the PCV model only if their joint distribution is quasisymmetric. Quasisymmetry requires that conditional on both observed bids being less than any arbitrary value $b$, the likelihood of a type $j$ bid being less than a type $k$ bid must be one half; otherwise the PCV model is rejected by the data. There currently exist no methods for performing this test over all levels of $b$ simultaneously, but tests at individual values of $b$ are easily performed with standard hypothesis testing techniques.

Under assumptions of bidder symmetry, affiliation and equilibrium bidding, Haile, Hong, and Shum (2003) develop a testing strategy for comparing hypotheses of common versus private values without the use of parametric assumptions. This method differs from other methods in that it doesn't test any particular PV or CV models (e.g., Paarsch (1992); Campo et al. (2003)); but rather, it enables testing of a null hypothesis including all PV models within the standard AV framework against an alternative including all CV models within that framework. These tests are developed for the case of exogenous bidder entry but they extend to settings of endogenous entry as well. Additionally, methods are provided to incorporate covariates into the testing procedure.

The tests they developed are based on theoretical results concerning the winner's curse. Recall that in equilibrium within the CV paradigm, the most optimistic signal of the unknown common 
value will lead to the winning bid. Hence, winning a CV auction entails receiving the potentially bad news that one had the most optimistic estimate of value. The magnitude of this bad news increases with the number of bidders. As argued by Wilson (1977), a rational bidder will anticipate this bad news, and adjust her expectation of the value of the object downward, as the number of bidders increases 11 This is in contrast to the APV information structure where a bidder's expectation of the value of the object is unaffected by their rivals' private information.

Therefore, Haile et al. proposed evaluating bidders' expected valuations in auctions with varying numbers of bidders. In a PV environment, these distributions should not vary with the number of bidders, whereas the CV alternative implies a first-order stochastic dominance relation. Of course, it is impossible to compare distributions of bidders' expectations, conditional on $\mathcal{N}$, so they relied on nonparametric methods developed by GPV, Li et al. $(2000,2002)$, as well as Hendricks et al. (2003) to compare empirical distributions of estimates of these expectations. First, under the null hypothesis of $\mathrm{PV}$, the nonparametric methods mentioned above are employed to estimate pseudoprivate values that underly each one of the bids. Second, three tests were proposed to evaluate the null hypothesis of PV against the alternative of CV. Two are tests for first-order stochastic dominance, one based on quantiles, and the second based on means. The final test computes a Kolmogorov-Smirnov statistic for distributional equivalence against the alternative of strict firstorder stochastic dominance.

Pinkse and Tan (2005) pointed out an important theoretical feature of the APV paradigm that must serve as a guide for future efforts to distinguish between private and common values information structures in first-price auctions. Prior to their work, it was believed that in general private values auctions, bids levels were strictly increasing in the number of potential bidders. It turns out however, that this is true only for the special case of IPV. Pinkse and Tan discovered a feature of general APV models which they call the affiliation effect. The affiliation effect is similar to the winner's curse, but is strategic in nature and has no bearing on the ex-post value of the sale object. Roughly, bidders realize that winning the auction reveals that competition was less fierce than a priori expected. Given the affiliation in their private information, they expect their opponents' values to be distributed roughly around their own. However, winning reveals that their opponents' values were all less, meaning that they were actually facing a set of opponents who were less aggressive than expected. Accordingly, they shade their bids by an additional margin in response. Moreover, just as with the winner's curse, the affiliation effect becomes greater as the number of bidders increases. Pinkse and Tan also showed that in first-price auctions with conditionally independent private values (a subcase of APV), equilibrium bids can actually be locally decreasing in the number of bidders, although at the upper bound of the support of values, bidding will still be monotonic. Thus, since bids in private value auctions may be (pointwise) nonmonotonic in the number of bidders, empirically distinguishing APV auctions from CV auctions may

\footnotetext{
${ }^{11}$ Granted, there is an additional competitive effect as number of bidders increases, which also drive bids upward; however the authors appealed to economic theory to separate these two effects, deriving estimates of the actual valuations given observations on bids, for the purpose of testing whether observed bids exhibit any significant response to the winner's curse. The interested reader is directed to the original work for further details.
} 
be more difficult than previously thought. Indeed these results imply that empirical observations of non-monotonic bidding (in $\mathcal{N}$ ) may only be taken as a rejection of the extreme IPV case, but not necessarily of other APV subcases.

Sareen (1999) is, to the best of our knowledge, the only example of Bayesian techniques being applied to the task of distinguishing private- and common-values models. Sareen's test is based on a comparison of posterior marginal likelihoods via the posterior odds ratio in order to see which specification the data favor most. Roughly, the posterior marginal likelihood under a given model indicates the degree of updating produced between specification of the prior distribution over model parameters and calculation of the posterior distribution after the data have been observed. The posterior odds ratio between two models is the ratio of their posterior marginal likelihoods. If equal prior probability is assumed for two models, $M$ and $M^{\prime}$, but $M$ has a significantly higher posterior marginal likelihood than $M^{\prime}$-in other words, if the posterior odds ratio of $M$ to $M^{\prime}$ is significantly greater than one - then the interpretation is that the learning from data that has taken place under $M$ was greater. Hence it can be said that the data favor model $M$ over $M^{\prime}$ or that $M$ was more informative as to the actual data generating process.

Sareen (1999) argued that there are distinct advantages to using the posterior odds ratio as a means of testing between pure common and independent private values. First, estimation and inference in many frequentist parametric auctions models is complicated by the fact that the support of the underlying distribution often depends on the model parameters, whereas a posterior odds ratio approach circumvents this problem. Second, a comparison of the polar extreme cases of PCV and IPV via the posterior odds ratio can potentially tell us something about their parent model, the general AV paradigm. If the posterior odds ratio is close to one, meaning that the data produces comparable learning in both models, then the researcher may conclude that elements of both are present in the actual data generating process, hence the appropriate structural model is affiliated values. If the posterior odds ratio or its reciprocal are close to zero, then the data reject the parent model in favor of one of the two polar cases. The one drawback to this method is that there is no well-defined criterion for establishing a threshold between significant differences between the posterior odds ratio and the benchmark values of one and zero.

\subsubsection{Testing for Affiliated Values}

A primary reason that so much attention has been given to the APV paradigm is because Milgrom and Weber (1982) showed that a unique equilibrium exists when the dependence between signals satisfies affiliation. Recently, de Castro (2010) demonstrated that weaker conditions are sufficient to guarantee existence and uniqueness of a monotone pure strategy equilibrium - specifically, such results are guaranteed as long as the inverse hazard rate is decreasing. Given most researchers impose affiliation, a natural question once weaker conditions can be used to guarantee uniqueness is whether the predictions of models with affiliation actually hold; for example, under affiliation the linkage principle provides a clear ranking of auction formats based on expected revenue which need not hold if affiliation in not satisfied. Such observations motivated de Castro and Paarsch (2010) 
who proposed testing for affiliation using grid distributions - a discretized version of a joint probability density function. Specifically, the authors allowed for an asymmetric private values model of a first-price auction, but considered in estimation bidders drawing valuations from the same marginal distribution due to data limitations. In estimation, they partitioned an unit hypercube $[0,1]^{\mathcal{N}}$ into $Y$ cells, normalized the observed bids to be between zero and one, and counted the number of $\mathcal{N}$-tuples that were observed in each cell. These quantities (counts) follow a multinomial distribution, the parameters of which can be estimated by standard methods. Thus, de Castro and Paarsch estimated the multinomial probabilities via ML under two scenarios: first, an unconstrained (standard) multinomial likelihood function and, second, a constrained likelihood function subject to constraints that the probabilities (parameters) satisfy affiliation. Their approach allowed the authors to use a likelihood ratio test to determine if affiliation was rejected for a given data sample. The authors considered the Michigan Department of Transportation data used by Hubbard et al. (forthcoming) and failed to reject a hypothesis of affiliation.

Jun, Pinkse, and Wan 2010) developed a test for affiliation which does not require the researcher to take a stance on the joint distribution of valuations, and is thus nonparametric. Moreover, the authors test is based off a mapping between the definition of affiliation and empirical distribution functions which do not require a bandwidth choice (as in kernel density estimation). However, the authors do advocate introducing an input parameter based on the sample size to obtain an asymptotically standard normal null distribution, which helps make inference easy. The input parameter essentially restricts an integral over weighted differences of products of indicator functions. Its choice involves a trade-off between optimal power of the test statistic, which suggests low values but involves lots of truncation, and a distorted size of the test for small values. The authors suggested choosing the input parameter so as to truncate ten percent of the observations in expectation; for this choice, the rejection frequency would be about 0.06 for a test of size 0.05 . Jun et al. investigated the choice of the input parameter and performance of the test in various Monte Carlo studies to better understand the behavior of their test statistic under the null hypothesis and the power of the test. Their experiments support their heuristic method for choosing the input parameter and the authors applied their test to a sample of OCS auctions, in which they found that bids were affiliated and that bids are affiliated with the number of bidders (both the actual and potential number of bidders).

While only one application was published in Jun et al. (2010), the authors considered three applications in a working version of their paper with the same title and failed to reject the hypothesis of affiliation between bids and the number of bidders in each case. The authors concluded that, "by far the most likely explanation for our results is that the number of bidders is endogenous." Li and Zhang (2010b) investigated affiliation within specific entry models and found that affiliation in the joint distribution of valuations or the joint distribution of entry costs leads to affiliation among potential bidders' entry decisions. They leveraged this implication to construct a test for affiliation (as well as asymmetry among potential bidders) based on a simulated ML estimation procedure of a multivariate probit model. Their test is based on entry behavior and does not use bidding 
information like most of the structural work we have discussed. Li and Zhang studied two entry models, one in which potential bidders draw private signals after an entry decision is made and one in which bidders draw private signals before deciding whether to enter the auction. Rather than test for the appropriate entry model, the authors note that both involve threshold rules: in the former model the entry cost of a bidder must be sufficiently low while in the latter the private signal must be sufficiently high. Affiliation in the entry costs or valuations make thresholds more stringent and lead to affiliation in the entry decisions. The authors then proposed reduced-form estimation of a binary latent variable model to explain the entry decision of a bidder in a given auction (after controlling for auction-specific and bidder-specific covariates as well as unobserved auction heterogeneity). Specifically, they assume the idiosyncratic errors follow a join normal distribution with zero mean but might have some positive correlation. Their hypothesis test is to see if this correlation is nonnegative (against a negative correlation alternative). To avoid multiple integrals which are computationally intensive they estimate the appropriate likelihood function via a simulated ML approach. The authors apply their test to a set of first-price, sealed-bid timber auctions conducted by the Oregon Department of Forestry and find that bidders are both affiliated and asymmetric.

\subsection{Auctions for Multiple Objects}

Up to this point, all of our discussion has focused on econometric methods developed for auctions of a single, indivisible good. We now conclude our survey by covering another important class of models which has received increasing attention in recent years by practitioners and researchers alike: auctions for multiple objects. We begin this section by introducing some key distinctions between auctions for multiple objects and their single-object cousins. This class of auctions is divided into two main categories: the simplest is called multi-unit auctions, or settings where multiple units of identical goods are sold; and the other is multi-object auctions, or settings where multiple distinct objects sold. The main difference between the two categories is that in the former, any set of objects is entirely characterized by it's cardinality, whereas in the latter, two sets containing the same number of goods may be valued differently to a given bidder. Therefore, in multi-unit auctions there is a well-defined ordering (common to all bidders) over all subsets of goods, whereas the same is not true, in general, for multi-object auctions (note also that the former is a special case of the latter). This distinction between the two settings also implies certain differences in the corresponding information structures. In the multi-unit setting where $L$ units are sold, private information held by bidder $i$ is usually modeled as a set of signals $\left(S_{i 1}, S_{i 2}, \ldots, S_{i L}\right)$ of the marginal valuations of each unit consumed, or in other words, bidder $i$ values a single unit at $S_{i 1}$, a bundle of two units at $S_{i 1}+S_{i 2}$, and so forth. In the multi-object setting where $L$ objects are sold, in order to allow for complementarity or substitutability of goods, bidder $i$ 's private information can be as complex as a complete set of signals $S_{i l}, l=1,2, \ldots, 2^{L}$, one each pertaining to the individual value of all $2^{L}$ possible bundles (subsets) of objects. In different situations, however, it may be appropriate to reduce this complexity by restricting the information structure in some way. In 
either case, one can easily imagine analogs to the information structures outlined in Section 2, which would determine whether private signals are correlated across bidders, and whether those signals represent idiosyncratic valuations of goods, or signals of common, but unknown valuations.

Concerning pricing rules, for multi-unit settings where items are simultaneously auctioned, bids come in the form of a demand schedule $p(y)$, which indicates the price at which a bidder would be willing to buy $y$ units. The two main pricing mechanisms that are employed in practice are the discriminatory auction and the uniform-price auction. Both mechanisms start by aggregating all bids into a single market demand curve. The former then awards the $y^{\text {th }}$ unit to the bidder whose individual demand was highest for that unit, (i.e., $p_{i}(y) \geq p_{j}(y), \forall j \neq i$ ), and sets a price according to that bidder's demand schedule. In that sense, the discriminatory format is the multiunit analog of the first-price auction. The uniform-price auction sets a single price $p^{*}$ according to the intersection of the market demand curve and the (fixed) supply line, making it the multiunit analog to the second-price auction. A final possibility is that the auctioneer may sequentially auction each unit off, one at a time, using any of the four canonical one-shot formats mentioned in the introduction. As for more general multi-object settings, pricing rules get a bit more complex and will be discussed below.

Before moving on, it is worth mentioning a few things about identification and estimation in multi-object settings. There are several reasons why these tasks become more challenging than in the single-object case. First of all, bidding strategies and private information are high-dimensional objects, and therefore more complex. In multi-unit auctions with $L$ goods for sale, bidders' private information and strategies are usually $L$-dimensional, and in multi-object auctions, they can be up to $2^{L}$-dimensional. Secondly, and as a result of this complexity, relatively little is known theoretically about equilibrium characterization, aside from existence. Often the first-order conditions of a bidder's decision problem are only necessary for optimality, unlike in the single-unit first-price auction, where they can be shown to be sufficient as well. This can often lead to various multi-object models being non-identified. Coping techniques for partial identification based on the first-order conditions (e.g., Cantillon and Pesendorfer (2007)), or more basic optimality conditions (e.g., Hortaçsu and McAdams (2010)) are discussed below.

A final practical challenge in auctions for multiple objects has to do with statistical and computational complexity, which arises both during estimation, as well as in computation of counterfactual experiments. Due to the high dimensionality of the observables, nonparametric kernel-smoothing techniques are less applicable for tasks like estimating distributions of bids and signals, as before. Kernel smoothing techniques, while flexible, suffer the curse of dimensionality: their statistical convergence rate slows rapidly (see Silverman (1986) for details) and the associated computational cost grows exponentially with the dimension of the distribution to be estimated. In practice, it is extremely rare for researchers to attempt kernel smoothing on any joint distribution of dimension three or higher.

Because of this difficulty, researchers have often chosen to employ a modified version of the GPV method, where costs are inferred from bids using some form of a necessary optimality condition, 
after which counterfactual exercises are based directly on the sample of estimated costs, rather than on their underlying distribution (e.g., see Hortaçsu and McAdams (2010) and Cantillon and Pesendorfer (2007)). Coping techniques for computing counterfactuals have also been developed, but as these are highly context-specific. Suffice it to say that significant progress has been made in auctions for multiple objects, but much work yet remains for future research. The various difficulties described above lead to tradeoffs of one form or another between model flexibility and tractability; these tradeoffs will be a central focus of the discussion in this section.

\subsubsection{Sequential Multi-Unit Auctions}

Donald, Paarsch, and Robert (2006) were among the first to analyze data on multi-unit auctions. They studied a setting in which $L$ units of a homogeneous good are auctioned off sequentially, with each individual unit priced identically as a one-shot English auction. Bidders are assumed to have private, multi-unit demands, and private information for player $i$ exists in the form of nonincreasing marginal valuations $S_{i 1} \geq S_{i 2} \geq \cdots \geq S_{i L}$. Despite the seemingly simple rules of this game, strategies available to players are quite complex because of the multi-unit nature of demand. For example, in a simple setting with two bidders and two units, bidder 1's decision of how to compete for the first unit balances several contingencies against one another. On the one hand, she could bid aggressively for the first unit, and have a chance to win both; on the other hand, she could choose not to compete for the first unit, meaning she would only have player 2's second valuation $S_{22} \leq S_{21}$ to compete with in the second round, possibly creating high surplus for that unit. One can easily imagine how strategies become even more complex in the general case with $\mathcal{N}$ bidders and $L$ units for sale.

Donald et al. solved this problem of intractability by showing that if bidders' valuations are symmetrically distributed in each round of the auction, then a simple, symmetric Bayes-Nash equilibrium can be characterized. Moreover, they derived a sufficient condition for symmetry to hold in each stage; namely, if the number of units valued by bidder $i$, call it $M_{i}$ is Poisson distributed, and all valuations are independent and identically distributed, or $S_{i m_{i}} \sim F_{S}(S), \forall m_{i}=$ $1, \ldots, M_{i}$ and $i=1, \ldots, \mathcal{N}$. As it turns out, this condition is empirically testable, as it implies that prices follow a submartingale process. However, estimation of the characterized equilibrium is still impeded by it's computational intractability 12 For this reason, Donald et al. proposed an empirical strategy similar to the simulation-based estimator of Laffont et al. (1995). Briefly, the stage-wise symmetry assumption also implies that the allocation mechanism is efficient, from which a multi-unit analog of the REP follows. To wit, under the above demand generation process, the sequential auction renders the same expected revenue to the seller as the generalized Vickrey auction, for which equilibrium strategies are trivial to compute: bidders truthfully report their marginal demand schedule $\left(S_{i 1}, \ldots, S_{i L}\right){ }^{13}$ Therefore, by specifying a parametric form for the

\footnotetext{
${ }^{12}$ See Donald et al. (2006, Section 3) for a full characterization of the equilibrium.

${ }^{13}$ The generalized Vickrey auction solicits sealed bids from bidders and then awards the $L$ objects to the bidders who submitted the $L$ highest bids. As for pricing, a bidder who wins $l_{i}$ units pays the $l_{i}$ highest losing bids, not including any losing bids player $i$ may have submitted herself.
} 
private value distribution, $F_{S}(s ; \boldsymbol{\theta})$, one can repeatedly simulate sale prices arising from equilibrium bidding in a generalized Vickrey auction, to calculate the expected prices that would arise in the sequential English format. In contrast to Laffont et al. (1995), Donald et al. incorporated these simulated prices into an empirical optimization routine based on the generalized method of moments (GMM), which seeks to choose values of $\boldsymbol{\theta}$ and the Poisson parameter to equate model-generated price moments with their empirical counterparts. They applied their structural estimator to data on multi-unit, sequential, English auctions of export permits for Russian timber.

Brendstrup (2007) re-examined the private-value, multi-unit, sequential, English auction setting, exploring alternative methods that may be employed when the stage-wise symmetry assumption of Donald et al. (2006) is inappropriate. Specifically, he attempted to identify and estimate a similar model as described above, but without the assumption that the number of units demanded by each bidder is Poisson distributed. Rather, all bidders simply draw $L$ independent and identically distributed signals from the private value distribution $F_{S}(S)$, and for each bidder the ordered signals constitute the marginal valuation schedule. The main innovation in this paper stems from the observation that, despite the strategic complexity arising in early stages of the auction, the final one is equivalent to a simple one-shot English auction, where bidders simply drop out once the price reaches the value of their highest remaining marginal value. Thus, Brendstrup used only data on price information in the final stage, and allocations from previous stages.

The main difficulty to overcome is that, even if bidders' marginal valuations are symmetrically distributed before bidding begins, those that remain in the final stage will be asymmetrically distributed, as they correspond to potentially different order statistics. Brendstrup's assumption that marginal valuations are given by an ordered random sample (along with an additional assumption pointed out by Lamy (2011) and discussed below) allowed for nonparametric identification of the model using results developed by Balakrishnan and Rao (1998) concerning distributions of order statistics from an independent but non-identically distributed sample. Even more remarkable (and once again taking into account additional concerns pointed out by Lamy (2011)) is the fact that model identification is possible even when bidders are ex-ante asymmetric, as when each valuation schedule is generated by an idiosyncratic distribution $F_{S_{i}}(S)$. In other words, it is possible to empirically disentangle fundamental asymmetry from that arising randomly as part of equilibrium play. These identification results set up a standard estimation strategy from second-price auctions in which the econometrician maps the distribution of last-stage prices into the latent valuation distribution nonparametrically, using the fact that the former can be expressed as a function of the latter.

Brendstrup and Paarsch (2006) built off Brendstrup (2007) and proposed a semi-nonparametric (SNP) approach to account for observable, auction-specific heterogeneity. Specifically, Brendstrup and Paarsch based their work on Gallant and Nychka (1987) who showed that any well-behaved distribution can be precisely expressed using an infinite sum of orthogonal basis polynomials. This implies that distributions may be estimated via ML using sums of polynomials truncated at some 
finite order that is allowed to grow without bound as the sample size increases 14 The advantage of SNP over kernel smoothing is that it allows for a tractable way to incorporate auction covariates $\mathbf{Z}$ into estimation. To wit, marginal valuations can be expressed as a flexible single index function

$$
S_{i}=\exp \left(\mathbf{Z} \boldsymbol{\beta}+U_{i}\right)
$$

where $\boldsymbol{\beta}$ is a vector of coefficients to be estimated and $U_{i}$ is a random idiosyncratic preference component for bidder $i$. By plugging this expression into the likelihood function in terms of signals $S$, the number of parameters to be estimated grows at a manageable rate with the number of covariates included. Both Brendstrup (2007) and Brendstrup and Paarsch (2006) (B\&BP) implemented their estimation schemes using data on sequential English fish auctions in Denmark.

In more recent work, Lamy (2011), building on theory developed by Katzman (1999), proved some important results regarding identification and estimation in sequential English auctions. As it turns out, one cannot be entirely agnostic about bidding behavior in early stages of the auction, as in B\&BP, or model identification fails and a selection bias will arise when estimating the underlying private value distribution using second-stage prices. Due to model complexity, Lamy focuses his analysis entirely on a two-stage, sequential English auction; for our purposes here, we shall illustrate the intuition behind the selection effect by considering only the two-bidder case. Recall that each bidder draws two independent valuations from a distribution $f_{S}$ with support $[0, \bar{s}]$, and then the ordered valuations become their schedule of marginal valuations. Call the high marginal valuation for player $i S_{1 i}$ and the low marginal valuation $S_{2 i}$. Ex ante, $S_{j i}$ has support $[0, \bar{s}]$ for each $j$ and $i$, and the low marginal valuation is also naturally bound by the constraint $S_{2 i} \leq S_{1 i}$ for each $i$. However, assume for a moment that first-round bidding is generated by a monotone function of each bidder's highest marginal valuation, and that bidder 1 wins the first round. Then the distributions of the remaining marginal valuations differ from the ex-ante distributions in the following ways: first, $S_{21}, S_{12}$, and $S_{22}$ all now have support [0, $S_{11}$ ], meaning $S_{12}$ is on average smaller than the ex-ante expectation. Second, $S_{22}$ is still subject to the additional constraint that $S_{22} \leq S_{12}$, whereas there are no additional constraints on values achieved by $S_{21}$, so it is on average higher than $S_{22}$. Note, however, that these arguments would change if first-round bidding was generated, for example, by a monotone function of the lowest marginal valuation, or by random bidding 15 Therefore, one cannot correct for possible differences between the ex-ante distribution (which affects allocations and prices in the first stage), and the conditional distributions (which determine the price in the second stage), without incorporating information from the bidding model of the first round of the auction.

This subtle insight has several important implications. First, even the simple two-stage version of the sequential English auction (with $\mathcal{N}$ bidders) is non-identified without assumptions on the

\footnotetext{
${ }^{14}$ When the support of the distribution is the entire real line, Hermite basis polynomials may be used, when it is half of the real line, the Laguerre basis applies, and when the support is compact, the Chebyshev basis applies. The SNP approach of Gallant and Nychka (1987) falls within a wider class of methods known as sieve estimators.

${ }^{15}$ Though it may seem odd to think about first-stage bidding as a function of each bidder's low marginal value, Katzman (1999) showed that there exist equilibria of the game consistent with this idea.
} 
bidding model in the first stage. In fact, even when the first-stage bidding model is specified, identification requires that $\mathcal{N} \geq 3$. Second, the nature of first-stage bidding will determine how to appropriately specify the distribution of the second-stage price. Lamy showed that B\&BP's estimators - based on the ex-ante distributions of marginal valuations - implicitly assume random bidding in the first stage in order for them to be unbiased. He also derived an expression for the second-stage price which corrects for the selection effect when bidding follows the monotone-inhigh-valuation rule, and demonstrated its superior performance relative to Brendstrup (2007) in a Monte Carlo experiment.

A final contribution of Lamy (2011) is in showing how estimation and even identification are aided when data on prices in both stages of the auction become available. First, he showed that if risk neutrality is assumed along with Bayes-Nash equilibrium bidding based on the highest marginal valuation, then the model is identified from observations of first-stage prices alone, and higher statistical precision can be achieved by incorporating this information into estimation as well. More significantly, Lamy also showed that observations of prices from both stages (along with a fully specified bidding model) allow one to relax the assumption (used by both Donald et al. (2006), Brendstrup (2007), and Brendstrup and Paarsch (2006)) that bidders' marginal valuations are order statistics from samples of independent draws from a common distribution $f_{S}$. Instead, one can identify a more general framework where bidders' valuations come from a general bivariate density $f_{\mathbf{S}}\left(S_{1}, S_{2}\right)$.

\subsubsection{Simultaneous Multi-Unit Auctions}

Février, Préget, and Visser (2004) is one of the earliest structural papers to study auctions within the sequential, multi-unit auction setting, and it is the only one of which we are aware to do so within a PCV information environment. In their model there is one unit of a perfectly divisible good with unknown value $V$. Bidders have symmetrically distributed private signals $S_{i} \sim F_{S \mid V}\left(S_{i} \mid V\right)$ of the true value of the good; alternatively, one could think of this model as representing many homogeneous goods whose individual marginal utilities are all constrained to be equal. Bidders are allowed to submit demand schedules dictating a complete schedule of prices at which they offer to buy a certain portion of the good. Février et al. proved parametric identification of the joint distribution of $(V, S)$, and estimated its parameters via a parametric version of the GPV method which reduces computational burden. Specifically, they begin by nonparametrically estimating the marginal distribution of the fraction of the good demanded, conditional on price and auction-specific covariates. Then, they plugged this estimate into the first-order conditions, which they showed are equivalent to a set of moment conditions relating the first-stage estimates to the parameters of the joint distribution of $(V, S)$. This leads to a conceptually simple estimator which they proved to be consistent and to have a well-behaved asymptotic distribution. Février et al. implemented this estimator on a sample of French Treasury auctions and found, in a counterfactual exercise, that the observed discriminatory pricing rule revenue dominates a hypothetical uniform rule by a substantial margin. 
Hortaçsu and McAdams (2010) was one of the first papers to study empirically a simultaneous auction mechanism for $L$ homogeneous goods within the IPV paradigm. Motivated by the example of Turkish treasury auctions, the authors specified private signals as representing a nonincreasing marginal value (NIMV) schedule $\mathbf{S}_{i}=\left(S_{i}(1), \ldots, S_{i}(L)\right)$, having a well-defined density over $\mathbb{R}^{L}$. Bids come in the form of a non-increasing price schedule, $\mathbf{p}_{i}(l)=\left(p_{i}(1), \ldots, p_{i}(L)\right)$, where $p_{i}(l)$ denotes the price offered by $i$ for the $l^{\text {th }}$ unit. The rules correspond to the discriminatory auction, in which the highest $L$ bids determine the price for each unit. The assumption of NIMV in various monetary auctions has both practical and technical motivations. On the practical side, profit maximizing banks that acquire short-term cash reserves will put them first to the most high-value use ${ }^{16}$ On the technical side, McAdams (2008) showed that private-value, multi-unit auctions are not identified in general, and deriving informative bounds on the distribution of private information may not be possible without the NIMV assumption.

Concerning Hortaçsu and McAdams (2010), perhaps the most notable aspect of this paper is that the authors were able to use the NIMV assumption to achieve nonparametric estimation with informative inferential power on a model with such high-dimensional private information. As mentioned above, a principal empirical difficulty in settings such as this is a lack of characterization of the theoretical equilibrium of the model. However, they were able to get considerable traction from the definition of a Bayes-Nash equilibrium: since all bidders are behaving optimally in equilibrium, an observed bid $p_{i}(l)$ must have rendered higher expected surplus than any other $p^{\prime} \neq p_{i}(l)$, given $i$ 's preferences and her competitors' strategies. Dropping the player subscript, if $\bar{p}>p(l)$ and all bidders submit strictly decreasing price schedules, this idea is expressed mathematically as

$$
[S(l)-p(l)] G[l ; p(l)] \geq[S(l)-\bar{p}] G(l ; \bar{p})
$$

where $G(l ; p)$ denotes one's probability of winning at least $l$ units in equilibrium with a bid of $p$ for the $l^{\text {th }}$ unit. This and the analogous condition for $\underline{p}<p(l)$ imply the following bounds on private values:

$$
p(l)+[p(l)-\underline{p}] \frac{G(l ; \underline{p})}{G[l ; p(l)]-G(l ; \underline{p})} \leq S(l) \leq p(l)+[\bar{p}-p(l)] \frac{G(l ; \bar{p})}{G(l ; \bar{p})-G[l ; p(l)]} .
$$

One can easily imagine replacing $p$ and $\bar{p}$ with observations on $p(l+1)$ and $p(l-1)$, respectively, from which a large enough sample size would eventually cause the bounds to collapse to a point. As the authors pointed out, in the single-unit case, as $\underline{p} \nearrow \bar{p}$ in expression (7) above, the result is exactly the same as equation (2) that GPV used to identify and estimate the canonical single-unit, first-price model.

An identification problem arises when bidders submit the same price offer for $k>1$ (consecutive) units, a commonly observed practice known as step bidding. In that case, the analogous version of (6) above might involve, say $S(l+1), S(l+2), \ldots, S(l+k)$ within a single inequality,

\footnotetext{
${ }^{16}$ Chapman, McAdams, and Paarsch (2006) put forth this argument in reference to the Bank of Canada's shortterm cash reserve auctions.
} 
which does not provide enough information to point identify $k$ separate private signals. It is still possible, however, to derive bounds similar to (7) which allow for identification of intervals to which a bidder's $l^{\text {th }}$ valuation must belong. The authors derived these bounds and estimated them nonparametrically using a simple resampling approach. Briefly, for a given bid $p_{i}(l)$, they used the next highest and lowest bids submitted by $i, p_{i}(l+k)$ and $p_{i}(l-j), k, j \geq 1$, and then estimated the corresponding win probabilities by repeatedly sampling from rival bids, with replacement, and computing the fraction of the time that $i$ would win at least $l,(l+k)$, and $(l-j)$ units. They showed how the procedure may be modified to incorporate (low-dimensional) covariates using kernel densities to weight sampled opponent bids, and demonstrated consistency of the resulting estimator of conditional win probabilities. Hortaçsu and McAdams implemented their method using Turkish treasury auction data. They were able to generate remarkably tight bounds for private valuations, and they used these bounds to perform a counterfactual revenue comparison to an alternative pricing rule, the uniform-price auction, which is known to induce truthful bidding in equilibrium. The results suggest that the discriminatory auction format dominates in terms of revenue and involves little efficiency loss, which provides a rationale for why it is widely favored among national treasury auctions 17

Kastl (2011) extended the model and resampling estimator of Hortaçsu and McAdams (2010) to explicitly model bidders' motivation for step bidding. Specifically, he considered two additions to the model: institutional constraints on the number of distinct price offers and costly bid formulation. In the case of bidding constraints, Kastl showed that, in equilibrium, bidders sometimes submit bids above marginal valuations for some units, if others falling under the same price offer are valued highly enough. He noted that this may cause Hortaçsu and McAdams (2010)'s estimator to underestimate the revenue generated by a uniform-price auction, although in his data on Czech treasury auctions, institutional bidding constraints are never observed to bind. The Czech treasury constrains bidders to submit a bid schedule with at most ten distinct price offerings. Kastl (2011) documented that the average price schedule involved roughly three steps, and the maximal number of steps in his sample was nine.

Another (seemingly more important) source of endogenous step bidding would arise if bidders had to pay a planning cost to formulate each additional pricing step into their demand schedule. Kastl derived bounds to partially identify both costs and marginal valuations from a sample of bids generated by a uniform-pricing mechanism. At the estimation stage, he employs a straightforward adaptation of Hortaçsu and McAdams (2010) resampling method. Kastl then compared the uniform-price auction to a hypothetical ideal mechanism which achieves both efficiency and full surplus extraction for the seller. His estimates suggested that in the case of the Czech treasury, the uniform price mechanism nearly accomplished both objectives.

A final word on model testability is worth mentioning here. One might be skeptical as to whether bidders in complex multi-unit auctions exhibit the required sophistication to engage in

\footnotetext{
${ }^{17}$ Thirty-nine out of forty-two countries surveyed by Bartolini and Cottarelli (1997) used the discriminatory auction mechanism in treasury auctions.
} 
the demanding best-response behavior required by Bayes-Nash equilibrium. In fact, several recent papers have attempted to answer this question by devising tests for best responding. The first was McAdams (2008), who proposed a test based on the fact that the analytic inverse-bid functions (the first-order conditions) do not restrict their nonparametric empirical counterparts to be monotone ${ }^{18}$ Chapman et al. (2006) implemented this test using data from Bank of Canada auctions for shortterm cash reserves. They found statistically significant deviations from best responding, but the economic significance of the deviations was minimal, suggesting that the Bayes-Nash equilibrium concept is a reasonable approximation to the real world. An alternative approach by Hortaçsu and Puller (2008), proposed nonparametric recovery of private information (as described above), followed by computation of best response to the observed bid distribution for each competitor. Using data on multi-unit procurement auctions for electricity generation in the State of Texas, they found that large firms (with large sums of money at stake) did remarkably well at best responding, while smaller firms' behavior was more prone to deviation from best responding.

\subsubsection{Multi-Object Auctions}

Cantillon and Pesendorfer (2007) studied a multi-object, simultaneous, first-price procurement auction with private costs. As usual, this procurement setting is intuitively similar to a private-value auction with diverse objects for sale. Before discussing identification and estimation of the model, it will be helpful to discuss some relevant theoretical nuances that come up in this setting. For simplicity of discussion, consider an auction for two contracts, call them $A$ and $B$, and denote the bundle of both contracts together as $A B$. All bidders submit sealed bids, and the auctioneer allocates and prices the contracts based on the combination of bids that minimize his total procurement cost 19 In addition, there may be cost synergies, meaning that a firm's cost of fulfilling contract $A B$ may be different from the combined costs for fulfilling $A$ and $B$ separately (in the private value case, this would be equivalent to different objects for sale being complementary goods). Because of this, an important policy consideration is whether to allow combination bidding, or submission of a single bid for the bundle $A B$, which may differ from the sum of bids on $A$ and $B$; bidding formats which allow for combination bidding are known as combinatorial auctions. If cost synergies are positive, meaning that total costs decrease when contracts are bundled, then combination bids will be strictly lower than the sum of the bids on individual contracts, or $b_{A B} \leq\left(b_{A}+b_{B}\right)$. It is easy to see that this can promote efficiency and lower procurement costs. However, combination bidding can also decrease efficiency as well, due to strategic bid leveraging which arises even in the absence of cost synergies. Briefly, if a combination bidder suspects that he has an advantage for contract $A$, he may leverage this advantage into a more aggressive bid for contract $B$ as he formulates his

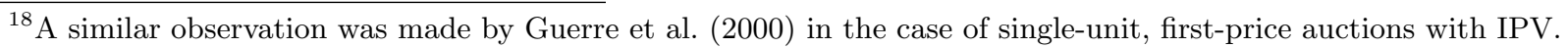

${ }^{19}$ Note that when combination bids are allowed, it need not be the case that the lowest bidder always wins a given item. For example, suppose there are three bidders, call them 1,2 , and 3 . Bidder 1 only values contract $A$, bidder 2 only values contract $B$ (their costs for fulfilling the other contract are prohibitively large), and bidder 3 values both. Suppose further that 1 bids $\$ 5$ for $A, 2$ bids $\$ 3$ for $B$, and 3 bids $\$ 3$ for $\mathrm{A}$ and $\$ 4$ for $B$ but only if contracts $A$ and $B$ are bundled together. Then 3 wins both contracts, despite overbidding player 2 on contract $B$.
} 
bid for the bundle $A B{ }^{20}$ In a sense, he is willing to make a lower profit margin on $B$ since his margin on $A$ is likely to be high. This can lead to inefficient contract allocation and even increased procurement costs in some cases.

Since bid leveraging puts downward pressure on combination bids, a structural bidding model is needed to empirically disentangle this effect from that of cost synergies. Moreover, if opponents' costs are highly correlated or if cost synergies are large, then strategic leveraging becomes less important, and combination bidding will still result in a welfare improvement; thus, a structural model can also provide useful policy prescriptions as well. However, identification and estimation are more difficult in this setting. First, bidding strategies are now determined by a system of equations - the first-order conditions of a bidder's decision problem - rather than a single equation, as in the canonical first-price auction. Moreover, the first-order conditions are now only necessary, but not sufficient for optimal behavior, having potentially multiple solutions. Cantillon and Pesendorfer showed that this system of equations can only nonparametrically identify a unique cost distribution if all bidders submit non-trivial bids (i.e., bids having a strictly positive probability of winning) for all contracts and combinations thereof. Otherwise, the model is partially identified, and the first-order conditions can be used to bound sets of cost distributions that are consistent with the data. For example, many equilibria of the model involve bidders not submitting bids on some contracts, in which case some of the bidders costs would only be set-identified.

Binding reserve prices are another source of under-identification, as in the canonical singleunit model, but there is yet another source in the multi-object case: restrictions on combination bidding. Specifically, if only individual bids $b_{A}$ or $b_{B}$ are allowed, or if the auction rules constrain combination bids so that $b_{A B} \leq\left(b_{A}+b_{B}\right)$, then it becomes more difficult for the econometrician to make inferences on valuations for bundles of contracts (i.e., cost synergies). The authors derived bounds on the underlying costs from the first-order conditions in each of the cases discussed above that can be used to partially identify the model.

As for estimation, Cantillon and Pesendorfer used a similar method to that of GPV, with adjustments to cope with the challenges of the multi-object environment. In the first stage, they estimated the bid distribution using a parametric approach, for two reasons. The first has to do with feasibility: nonparametric kernel smoothing methods, while flexible, are subject to the curse of dimensionality, as the associated computational cost grows exponentially in the dimension of the distribution to be estimated. In a multi-object auction, where bidders have separate bids for each contract and each distinct bundle of contracts, this problem quickly becomes pronounced. Moreover, the authors also conditioned the bid distribution on contract and bidder covariates, which further increases the dimensionality of the joint distribution to be estimated. The second reason for using parametric methods is statistical: as illustrated by Silverman (1986), kernel-

\footnotetext{
${ }^{20}$ A similar type of strategic bid distortion was recognized by Athey and Levin (2001) at "scale sales" held by the U.S. Forest Service. At these auctions, the Forest Service announces the quantity of each species of tree it expects on a tract of timber. A bid is a sum, over the various species, of the per-unit bids submitted multiplied by the quantities announced. The highest bidder wins the right to harvest the timber. Since the winner pays based on actual harvests and given the rates specified in the bidding process, bidders have incentive to skew their bids by overbidding on species that they feel were underestimated.
} 
smoothed density estimates require more data than parametric methods to obtain precise estimates, and the data requirement quickly increases with the dimensionality of the distribution. Thus, parametric methods can help to not only reduce computational burden - which usually grows with dimensionality at a polynomial rate - but also to cope with a relatively small sample size. Cantillon and Pesendorfer specified the joint distribution of bids (conditional on covariates) as a multivariate lognormal distribution and estimated its parameters by the method of simulated moments.

Just as in the canonical GPV method, their second stage involves inferring costs from bids, using the first-order conditions in conjunction with the estimated bid distribution. Recall that their primary research question involved evaluating the nature and importance of cost synergies and cost correlation across firms, so as to characterize the welfare implications of allowing combination bidding. As this is the case, there is no need to infer cost distributions from the data, only costs. However, if such a need arose, the researcher would have to employ a flexible (i.e., nonparametric or semi-nonparametric) estimator so as to maintain internal consistency within the model. Of course, such an undertaking would be subject to the difficulties described above.

Cantillon and Pesendorfer applied their estimation scheme to a procurement auction for public transportation contracts (bus routes) in London, England. Despite the difficulties of partial identification, they were able to estimate that the median cost synergy is actually negative, implying decreasing returns to scale for bussing firms. They also found that more than half of the contracts were awarded to someone other than the low-cost bidder (due to strategic bid leveraging), and that welfare could significantly increase by allowing bids on individual stand-alone contracts only.

To date, Cantillon and Pesendorfer is the only paper of which we are aware that performs structural estimation of a combinatorial auction; however, some caveats are worth mentioning. First, the authors are aided, computationally, by the relatively small size of the London bus route auctions, in which at most three separate contracts are up for bids at any one time. The only other paper of which we are aware, currently, that attempts an empirical analysis of combinatorial auctions is Epstein, Olivares, Weintraub, and Yung (2011). Specifically, the authors studied the same policy questions as Cantillon and Pesendorfer, in a similar (but much larger scale) setting in which many school-lunch supply contracts are simultaneously auctioned, and bidders are allowed to submit bids for packages of up to eight contracts. Some firms in their data set submit thousands of bids in a single auction. Because of the resulting computational complexity, Epstein et al. constructed a flexible reduced-form empirical model which attempts to estimate costs from bids while empirically disentangling the theoretical influences of cost synergies and strategic contract bundling. The reduced-form estimation precludes computation of counterfactuals, however the authors are still able to make policy recommendations based on their findings. With auctions becoming an increasingly important supply-chain procurement tool, further research is needed to make structural estimation feasible in larger settings. 


\section{Conclusion}

In this survey, we have detailed structural econometric research that has been developed to study various types of auctions. We have chosen to organize major contributions by partitioning the AV model into subcategories based on commonly-used informational structures in hopes of complementing the book-length treatments of this topic in Paarsch and Hong (2006) or Athey and Haile (2007), who partitioned the literature by different auction formats. To our knowledge, no one has formally tied together the informational paradigms studied in empirical work in a unified way that is consistent with theoretical research. While our discussion of any one piece of research is no substitute for a thorough reading of the original work (we did not intend it to be), we have tried to provide enough insight into each piece of research we discussed so that readers can understand the fundamental contributions and have an understanding of how it fits in with previous and subsequent research. We think our discussions of the fundamental information paradigms not only provide a good introduction to researchers new to auctions and/or structural econometric methods, but that they also provide a perspective that will appeal to experienced researchers as well. We have also included an overview of several special topics that we hope are helpful to researchers either trying to allow for one of these extensions to a core model or trying to improve upon the current approaches. A quick scan through the text of our special topics section shows that most research in these areas has taken place in the past few years. Our view is that these areas involve not only interesting topics, but that there are many interesting extensions and developments to come.

\section{References}

Akerlof, G. A. (1970): "The Market for 'Lemons': Quality Uncertainty and the Market Mechanism," Quarterly Journal of Economics, 84, 488-500.

Amemiya, T. (1985): Advanced Econometrics, Harvard University Press.

An, Y., Y. Hu, and M. Shum (2010): "Estimating First-Price Auctions with an Unknown Number of Bidders: A Misclassification Approach," Journal of Econometrics, 157, 328-341.

Aradillas-López, A., A. Gandhi, and D. Quint (2011): "Identification and Testing in Ascending Auctions with Unobserved Heterogeneity," Working paper, University of Wisconsin.

Athey, S. (2001): "Single Crossing Properties and the Existence of Pure Strategy Equilibria in Games of Incomplete Information," Econometrica, 69, 861-889.

Athey, S. and P. A. Haile (2002): "Identification of Standard Auction Models," Econometrica, 70, $2107-2140$.

Athey, S. and P. A. Haile (2007): "Nonparametric Approaches to Auctions," Handbook of Econometrics, 6, 3847-3965.

Athey, S. and J. Levin (2001): "Information and Competition in U.S. Forest Service Timber Auctions," Journal of Political Economy, 109, 375-417. 
Athey, S., J. Levin, and E. Seira (2011): "Comparing Open and Sealed Bid Auctions: Evidence from Timber Auctions," Quarterly Journal of Economics, 126, 207-257.

Bajari, P. and A. Hortaçsu (2003): "The Winner's Curse, Reserve Prices, and Endogenous Entry: Empirical Insights from eBay Auctions," RAND Journal of Economics, 34, 329-355.

Balakrishnan, N. and C. Rao (1998): Handbook of Statistics, Elsevier, volume 16. Order Statistics: Theory and Methods.

Bartolini, L. and C. Cottarelli (1997): "Treasury bill auctions: Issues and uses," in M. I. Blejer and T. Ter-Minassian, eds., Macroeconomic Dimensions of Public Finance: Essays in Honor of Vito Tanzi, London: Routledge, 267-336.

Bikhchandani, S., P. A. Haile, and J. G. Riley (2002): "Symmetric Separating Equilibria in English Auctions," Games and Economic Behavior, 38, 19-27.

Brendstrup, B. (2007): "Nonparametric Estimation of Sequential English Auctions," Journal of Econometrics, 141, 460-481.

Brendstrup, B. and H. J. Paarsch (2006): "Identification and Estimation in Sequential, Asymmetric, English Auctions," Journal of Econometrics, 134, 69-94.

Campo, S., E. Guerre, I. Perrigne, and Q. Vuong (2011): "Semiparametric Estimation of First-Price Auctions with Risk Averse Bidders," The Review of Economic Studies, 78, 112-147.

Campo, S., I. Perrigne, and Q. Vuong (2003): "Asymmetry in First-Price Auctions with Affiliated Private Values," Journal of Applied Econometrics, 18, 179-207.

Cantillon, E. and M. Pesendorfer (2007): "Combination Bidding in Multi-Unit Auctions," Working paper, Center for Economic Policy Research.

Chapman, J. T. E., D. McAdams, and H. J. Paarsch (2006): "Bounding Best-Response Violations in Discriminatory Auctions with Private Values," Working paper, Bank of Canada.

Chernozhukov, V. and H. Hong (2004): "Likelihood Estimation and Inference in a Class of Nonregular Econometric Models," Econometrica, 72, 1445-1480.

Cox, D. R. (1961): “Tests of Separate Families of Hypotheses (Proc. 4th Berkeley Symp.)," Math. Statist. Prob., 1, 105-123.

Cox, D. R. (1962): "Further Results on Tests of Separate Families of Hypotheses," Journal of the Royal Statistical Society. Series B (Methodological), 24, 406-424.

de Castro, L. (2010): “Affiliation, Equilibrium Existence and Revenue Ranking of Auctions," Working paper, Northwestern University.

de Castro, L. I. and H. J. Paarsch (2010): "Testing Affiliation in Private-Values Models of FirstPrice Auctions Using Grid Distributions," Annals of Applied Statistics, 4, 2073-2098.

Donald, S. G. and H. J. Paarsch (1993): "Piecewise Pseudo-Maximum Likelihood Estimation in Empirical Models of Auctions," International Economic Review, 34, 121-148.

Donald, S. G. and H. J. Paarsch (1996): "Identification, Estimation, and Testing in Parametric Empirical Models of Auctions within the Independent Private Values Paradigm," Econometric Theory, 12, 517-567. 
Donald, S. G. and H. J. Paarsch (2002): "Superconsistent Estimation and Inference in Structural Econometric Models Using Extreme Order Statistics," Journal of Econometrics, 109, 305-340.

Donald, S. G., H. J. Paarsch, and J. Robert (2006): "An Empirical Model of the Multi-Unit, Sequential, Clock Auction," Journal of Applied Econometrics, 21, 1221-1247.

Elyakime, B., J. J. Laffont, P. Loisel, and Q. Vuong (1994): "First-Price Sealed-Bid Auctions with Secret Reservation Prices," Annales d'Economie et de Statistique, 115-141.

Elyakime, B., J. J. Laffont, P. Loisel, and Q. Vuong (1997): "Auctioning and Bargaining: An Econometric Study of Timber Auctions with Secret Reservation Prices," Journal of Business \& Economic Statistics, 15, 209-220.

Epstein, R., M. Olivares, G. Y. Weintraub, and D. Yung (2011): "The Design of Combinatorial Auctions for Procurement: An Empirical Study of the Chilean School Meals Auction," Working paper, Columbia Business School.

Février, P., R. Préget, and M. Visser (2004): "Econometrics of Share Auctions," Working paper, University of Chicago.

Flambard, V. and I. Perrigne (2006): "Asymmetry in Procurement Auctions: Evidence from Snow Removal Contracts," The Economic Journal, 116, 1014-1036.

Gallant, A. R. and D. W. Nychka (1987): "Semi-Nonparametric Maximum Likelihood Estimation," Econometrica, 55, 363-390.

Govindan, S. and R. Wilson (2010a): "Existence of Equilibria in Auctions with Interdependent Values," Working paper, Stanford Graduate School of Business.

Govindan, S. and R. Wilson (2010b): "Existence of Equilibria in Private-Value Auctions," Working paper, Stanford Graduate School of Business.

Guerre, E., I. Perrigne, and Q. Vuong (2000): "Optimal Nonparametric Estimation of First-Price Auctions," Econometrica, 68, 525-574.

Guerre, E., I. Perrigne, and Q. Vuong (2009): "Nonparametric Identification of Risk Aversion in First-Price Auctions under Exclusion Restrictions," Econometrica, 77, 1193-1227.

Haile, P. A., H. Hong, and M. Shum (2003): "Nonparametric tests for common values at first-price sealed-bid auctions," NBER Working Paper.

Haile, P. A. and E. Tamer (2003): "Inference with an Incomplete Model of English Auctions," Journal of Political Economy, 111, 1-51.

Harsanyi, J. (1967): "Games with Incomplete Information Played by Bayesian Players, Parts 1-3," Management Science, 14.

Hausman, J. A. (1978): "Specification Tests in Econometrics," Econometrica, 46, 1251-1271.

Hendricks, K., J. Pinkse, and R. H. Porter (2003): "Empirical Implications of Equilibrium Bidding in First-Price, Symmetric, Common Value Auctions," The Review of Economic Studies, 70, 115145 . 
Hickman, B. R. and T. P. Hubbard (2012): "What Happens When You Can Keep the Data: Replacing Sample Trimming with Boundary Correction in Nonparametric Estimation of FirstPrice Auctions," Working paper, University of Chicago.

Hickman, B. R., T. P. Hubbard, and H. J. Paarsch (2011): "Investigating the Economic Importance of Pricing-Rule Mis-Specification in Empirical Models of Electronic Auctions," Working paper, University of Chicago.

Hirano, K. and J. R. Porter (2003): "Asymptotic Efficiency in Parametric Structural Models with Parameter-Dependent Support," Econometrica, 71, 1307-1338.

Hong, H. and M. Shum (2002): "Increasing Competition and the Winner's Curse: Evidence from Procurement," The Review of Economic Studies, 69, 871-898.

Hong, H. and M. Shum (2003): "Econometric Models of Asymmetric Ascending Auctions," Journal of Econometrics, 112, 327-358.

Hortaçsu, A. and D. McAdams (2010): "Mechanism Choice and Strategic Bidding in Divisible Good Auctions: An Empirical Analysis of the Turkish Treasury Auction Market," Journal of Political Economy, 118, 833-865.

Hortaçsu, A. and S. L. Puller (2008): "Understanding Strategic Bidding in Multi-Unit Auctions: a Case Study of the Texas Electricity Spot Market," RAND Journal of Economics, 39, 86-114.

Hu, Y., D. McAdams, and M. Shum (2010): "Nonparametric Identification of First-Price Auctions with Non-Separable Unobserved Heterogeneity," Working paper, Duke University.

Hubbard, T. P., T. Li, and H. J. Paarsch (forthcoming): "Semiparametric Estimation in Models of First-Price, Sealed-Bid Auctions with Affiliation," Journal of Econometrics.

Hubbard, T. P. and H. J. Paarsch (2011): "On the Numerical Solution of Equilibria in Auction Models with Asymmetries within the Private-Values Paradigm," Working paper, Texas Tech University.

Jackson, M. O., L. K. Simon, J. M. Swinkels, and W. R. Zame (2002): "Communication and Equilibrium in Discontinuous Games of Incomplete Information," Econometrica, 70, 1711-1740.

Jackson, M. O. and J. M. Swinkels (2005): "Existence of Equilibrium in Single and Double Private Value Auctions," Econometrica, 73, 93-139.

Ji, L. and T. Li (2008a): "Multi-Round Procurement Auctions with Secret Reserve Prices: Theory and Evidence," Journal of Applied Econometrics, 23, 897-923.

Ji, L. and T. Li (2008b): "Multi-Round Procurement Auctions with Secret Reserve Prices: Theory and Evidence," Journal of Applied Econometrics, 23, 897-923.

Jofre-Bonet, M. and M. Pesendorfer (2003): "Estimation of a Dynamic Auction Game," Econometrica, 71, 1443-1489.

Jun, S. J., J. Pinkse, and Y. Wan (2010): "A Consistent Nonparametric Test of Affiliation in Auction Models," Journal of Econometrics, 159, 46-54.

Kastl, J. (2011): "Discrete Bids and Empirical Inference in Divisible Good Auctions," The Review of Economic Studies. 
Katzman, B. (1999): "A Two Stage Sequential Auction with Multi-Unit Demands," Journal of Economic Theory, 86, 77-99.

Komarova, T. (2011): "Partial Identification in Asymmetric Second-Price Auctions in the Absence of Independence," Working paper, London School of Economics.

Krasnokutskaya, E. (2011): "Identification and Estimation of Auction Models with Unobserved Heterogeneity," The Review of Economic Studies, 78, 293-327.

Krasnokutskaya, E. and K. Seim (forthcoming): "Bid Preference Programs and Participation in Procurement Auctions," The American Economic Review.

Krishna, V. (2002): Auction Theory, Academic Press.

Laffont, J. J., H. Ossard, and Q. Vuong (1995): "Econometrics of First-Price Auctions," Econometrica, 953-980.

Laffont, J. J. and Q. Vuong (1996): "Structural Analysis of Auction Data," The American Economic Review, 86, 414-420.

Lamy, L. (2011): "Identification and Estimation of Sequential English Auctions," Working paper, Paris School of Economics.

Lamy, L. (forthcoming): "The Econometrics of Auctions with Asymmetric Anonymous Bidders," Journal of Econometrics.

Lebrun, B. (1996): "Existence of an Equilibrium in First Price Auctions," Economic Theory, 7, 421-443.

Lebrun, B. (1999): "First Price Auctions in the Asymmetric N Bidder Case," International Economic Review, 40, 125-142.

Lebrun, B. (2006): "Uniqueness of the Equilibrium in First-Price Auctions," Games and Economic Behavior, 55, 131-151.

Lewis, G. (2011): "Asymmetric Information, Adverse Selection and Online Disclosure: The Case of eBay Motors," The American Economic Review, 101, 1535-1546.

Li, T. (2005): "Econometrics of first-price auctions with entry and binding reservation prices," Journal of Econometrics, 126, 173-200.

Li, T. and I. Perrigne (2003): "Timber Sale Auctions with Random Reserve Prices," Review of Economics and Statistics, 85, 189-200.

Li, T., I. Perrigne, and Q. Vuong (2000): "Conditionally Independent Private Information in OCS Wildcat Auctions," Journal of Econometrics, 98, 129-161.

Li, T., I. Perrigne, and Q. Vuong (2002): "Structural Estimation of the Affiliated Private Value Auction Model," RAND Journal of Economics, 33, 171-193.

Li, T., I. Perrigne, and Q. Vuong (2003): "Semiparametric Estimation of the Optimal Reserve Price in First-Price Auctions," Journal of Business and Economic Statistics, 21, 53-64.

Li, T. and Q. Vuong (1998): "Nonparametric Estimation of the Measurement Error Model Using Multiple Indicators," Journal of Multivariate Analysis, 65, 139-165. 
Li, T. and B. Zhang (2010a): "Affiliation and Entry in First-Price Auctions with Heterogeneous Bidders," Working paper, Vanderbilt University.

Li, T. and B. Zhang (2010b): "Testing for Affiliation in First-Price Auctions using Entry Behavior," International Economic Review, 51, 837-850.

Li, T. and X. Zheng (2009): "Entry and Competition Effects in First-Price Auctions: Theory and Evidence from Procurement Auctions," The Review of Economic Studies, 76, 1397-1429.

Li, T. and X. Zheng (forthcoming): "Information Acquisition and/or Bid Preparation: A Structural Analysis of Entry and Bidding in Timber Sale Auctions," Journal of Econometrics.

Lizzeri, A. and N. Persico (2000): "Uniqueness and Existence of Equilibrium in Auctions with a Reserve Price," Games and Economic Behavior, 30, 83-114.

Lu, J. and I. Perrigne (2008): "Estimating risk aversion from ascending and sealed-bid auctions: The case of timber auction data," Journal of Applied Econometrics, 23, 871-896.

MacKinnon, J., H. White, and R. Davidson (1983): "Tests for Model Specification in the Presence of Alternative Hypotheses: Some Further Results," Journal of Econometrics, 21, 53-70.

Maskin, E. and J. Riley (1984): "Optimal Auctions with Risk Averse Buyers," Econometrica, 52, $1473-1518$.

Maskin, E. and J. Riley (2000a): "Asymmetric Auctions," The Review of Economic Studies, 67, 413-438.

Maskin, E. and J. Riley (2000b): "Equilibrium in Sealed High Bid Auctions," The Review of Economic Studies, 67, 439-454.

Maskin, E. and J. Riley (2003): "Uniqueness of Equilibrium in Sealed High-Bid Auctions," Games and Economic Behavior, 45, 395-409.

McAdams, D. (2008): "Partial Identification and Testable Restrictions in Multi-Unit auctions," Journal of Econometrics, 146, 74-85.

McAfee, R. P. and D. Vincent (1992): "Updating the Reserve Price in Common-Value Auctions," The American Economic Review, 82, 512-518.

McFadden, D. (1996): “Lectures on Simulation-Assisted Statistical Inference. ec-squared conference, florence, italy, december 12, 1996," Working paper, University of California-Berkeley.

Milgrom, P. (2004): Putting Auction Theory to Work, Cambridge University Press.

Milgrom, P. R. and R. J. Weber (1982): "A Theory of Auctions and Competitive Bidding," Econometrica, 50, 1089-1122.

Myerson, R. B. (1981): "Optimal Auction Design," Mathematics of Operations Research, 6, 55-73.

Nelsen, R. (1999): An Introduction to Copulas, Springer.

Paarsch, H. J. (1992): "Deciding between the Common and Private Value Paradigms in Empirical Models of Auctions," Journal of Econometrics, 51, 191-215. 
Paarsch, H. J. (1997): "Deriving an Estimate of the Optimal Reserve Price: An Application to British Columbian Timber Sales," Journal of Econometrics, 78, 333-357.

Paarsch, H. J. and H. Hong (2006): An Introduction to the Structural Econometrics of Auction Data, The MIT Press.

Pinkse, J. and G. Tan (2005): "The Affiliation Effect in First-Price Auctions," Econometrica, 73, $263-277$.

Reny, P. J. (1999): "On the Existence of Pure and Mixed Strategy Nash Equilibria in Discontinuous Games," Econometrica, 67, 1029-1056.

Reny, P. J. and S. Zamir (2004): "On the Existence of Pure Strategy Monotone Equilibria in Asymmetric First-Price Auctions," Econometrica, 72, 1105-1125.

Riley, J. G. and W. F. Samuelson (1981): "Optimal Auctions," The American Economic Review, 71, 381-392.

Roberts, J. W. (2011): "Unobserved Heterogeneity and Reserve Prices in Auctions," Working paper, Duke University.

Roth, A. E. and A. Ockenfels (2002): "Last-Minute Bidding and the Rules for Ending Second-Price Auctions: Evidence from eBay and Amazon Auctions on the Internet," The American Economic Review, 92, 1093-1103.

Sailer, K. (2006): "Searching the eBay Marketplace," Working paper, University of Munich.

Sareen, S. (1999): "Posterior Odds Comparison of a Symmetric Low-Price, Sealed-Bid Auction within the Common-Value and the Independent-Private-Values Paradigms," Journal of Applied Econometrics, 14, 651-676.

Silverman, B. W. (1986): Density Estimation for Statistics and Data Analysis, London: Chapman and Hall.

Song, U. (2004a): "Nonparametric Estimation of an eBay Auction Model with an Unknown Number of Bidders," Working paper, University of British Columbia.

Song, U. (2004b): "Nonparametric Identification and Estimation of a First-Price Auction Model with an Uncertain Number of Bidders," Working paper, University of British Columbia.

Thiel, S. E. (1988): "Some evidence on the winner's curse," The American Economic Review, 78, 884-895.

Vickrey, W. (1961): "Counterspeculation, auctions, and competitive sealed tenders," Journal of Finance, 16, 8-37.

White, H. (1982): "Regularity Conditions for Cox's Test of Non-Nested Hypotheses," Journal of Econometrics, 19, 301-318.

Wilson, R. (1977): "A Bidding Model of Perfect Competition," The Review of Economic Studies, $44,511-518$.

Wilson, R. (1979): "Auctions of Shares," Quarterly Journal of Economics, 93, 675-689. 
Wilson, R. (1998): "Sequential Equilibria of Asymmetric Ascending Auctions: The Case of LogNormal Distributions," Economic Theory, 12, 433-440.

Zheng, X. (2009): "Quantifying the cost of excess market thickness in timber sale auctions," International Journal of Industrial Organization, 27, 553-566.

\section{A Existence and Uniqueness}

In Table 1, we present a list of important theoretical results on existence and uniqueness in auctions. Structural research requires such properties to argue that the data generating process corresponds to a particular model of bidding.

\begin{tabular}{|c|c|}
\hline Reference & Result \\
\hline Vickrey (1961) & $\begin{array}{l}\text { Existence of dominant strategy equilibrium of ascending-price auc- } \\
\text { tion within IPV paradigm }\end{array}$ \\
\hline Wilson (1977) & $\begin{array}{l}\text { Sufficient conditions for existence of equilibrium in sealed-bid first- } \\
\text { price auction when private signals are i.i.d., conditional on an un- } \\
\text { known common component }\end{array}$ \\
\hline Milgrom and Weber (1982) & $\begin{array}{l}\text { Existence of equilibrium in sealed-bid first-price, second-price and } \\
\text { ascending-price auctions under AV }\end{array}$ \\
\hline Maskin and Riley $(1984)$ & $\begin{array}{l}\text { Existence and uniqueness of equilibrium in first-price and second- } \\
\text { price auctions when bidders are risk averse }\end{array}$ \\
\hline \begin{tabular}{|l|l|l|} 
Lebrun & 1996,1999 & $2006)$ \\
\end{tabular} & $\begin{array}{l}\text { Existence and uniqueness of equilibrium in first-price auctions } \\
\text { when bidders are potentially asymmetric }\end{array}$ \\
\hline Maskin and Riley $(2000 \mathrm{~b})$ & $\begin{array}{l}\text { Existence of a monotonic equilibrium in the first-price auction when } \\
\text { bidders have (potentially asymmetric) affiliated private values and } \\
\text { Von Neumann-Morgenstern preferences }\end{array}$ \\
\hline $\begin{array}{l}\text { Bikhchandani, Haile, and Riley } \\
(2002)\end{array}$ & $\begin{array}{l}\text { Existence and non-uniqueness of separating equilibria in English } \\
\text { auctions with affiliated values }\end{array}$ \\
\hline Maskin and Riley (2003) & $\begin{array}{l}\text { Uniqueness of a monotonic equilibrium in the first-price auction } \\
\text { under the conditions that: (i) bidders have (a)symmetric APV and } \\
\text { VNM preferences; (ii) absolute risk aversion is non-increasing; (iii) } \\
\text { the supports of the different buyers' distributions of values have } \\
\text { the same upper endpoint. }\end{array}$ \\
\hline $\begin{array}{l}\text { Reny and Zamir } 2004) \\
\text { Reny }(1999)\end{array}$ & $\begin{array}{l}\text { Existence of a monotonic equilibrium in first-price auctions } \\
\text { with asymmetric bidders having affiliated common values }\end{array}$ \\
\hline $\begin{array}{l}\text { Lizzeri and Persico }(2000) \\
\text { Athey }(2001) \\
\text { Jackson, Simon, Swinkels, and Zame } \\
\text { (2002) } \\
\text { Jackson and Swinkels }(2005) \\
\end{array}$ & $\begin{array}{l}\text { Existence of pure and mixed equilibria in general games of } \\
\text { incomplete information, including many exotic types of auctions } \\
\text { with non-standard pricing rules, including some results on } \\
\text { existence of monotonic equilibria (see Jackson and Swinkels } \\
(2005) \text { ) }\end{array}$ \\
\hline $\begin{array}{l}\text { Govindan and Wilson } 2010 \mathrm{a} \text { b }) \\
\text { de Castro }(2010)\end{array}$ & $\begin{array}{l}\text { Investigate conditions weaker than affiliation which separately } \\
\text { guarantee existence, uniqueness, and pure monotonic equilibria. }\end{array}$ \\
\hline Katzman $(1999)$ & $\begin{array}{l}\text { Efficiency and revenue equivalence for sequential, second-price auc- } \\
\text { tions with two rounds and based on the link between complete and } \\
\text { incomplete information auction games. }\end{array}$ \\
\hline
\end{tabular}

Table 1: Existence and Uniqueness of Equilibrium in Auctions 